\title{
Free vibration analysis of truncated conical fiber metal laminate (FML) shells
}

\author{
Faramarz Ashenai Ghasemi ${ }^{1, a}$, Reza Ansari ${ }^{2}$ and Rahim Bakhodai Paskiabi ${ }^{1}$ \\ 1 Department of Mechanical Engineering, Shahid Rajaee Teacher Training University (SRTTU), \\ 16788-15811 Lavizan, Tehran, Iran \\ 2 Department of Mechanical Engineering, University of Guilan, P.O. Box 3756, Rasht, Iran
}

Received 29 June 2013, Accepted 25 October 2013

\begin{abstract}
In this paper, an analytical solution is developed for free vibration analysis of conical fiber metal shells. In order to find constitutive relations, the assumptions of thins hells are used and the governing equations are based on Love's theory. The Galerkin method is employed to solve the governing equations in which beam functions are used to approximate the mode shapes. Using beam functions enables us to assess the effects of different boundary conditions on the frequency response of the shells. Numerical comparisons of the present and previously published results confirm the accuracy of the current approach. Additionally, the influences of geometrical parameters and embedding aluminum plies in different layers of the structure on natural frequency of the conical shells with various boundary conditions are investigated. It can be observed that the more the aluminum plies are used, the greater natural frequency of the structure will be reached. Except the clamped-free boundary conditions, the results also indicate that if the aluminum plies are embedded in the top and bottom layers of the laminate, natural frequency reaches its maximum value.
\end{abstract}

Key words: Conical shell / free vibration / Galerkin method / beam function

\section{Introduction}

Among different types of shells, truncated conical shells have different applications in spacecrafts, robots, tanks and other mechanical structures. Up to now, the mechanical behavior of these structures has been investigated by many researchers under various loadings. In recent years, many studies have numerically worked on mechanical behavior of conical shells experimentally or analytically.

Wilkins et al. [1] investigated the free vibrations of orthotropic sandwich conical shells under different boundary conditions. Ramsey [2] studied elastic and plastic buckling of conical shells under axial compressive loads. Vibrational properties of joined conical-cylindrical shells were analyzed by Irie et al. [3]. Liu and Zhou [4] presented buckling analysis of orthogonal anisotropic circular conical shells under uniformly external pressure in the elastic regime. Thambiratnam and Zhuge [5] applied the finite element method for investigating axial symmetric free vibrations of conical shells with variable thickness and found that frequencies decreased with the apex angle

\footnotetext{
${ }^{a}$ Corresponding author:

Faramarz_ashenai_ghasemi@yahoo.com
}

and increased with the $R / H$ ratio, gradient of the wall slope and number of slopes.

Shu [6] used a numerical method for the analysis of isotropic conical shells. Elastic buckling and the after-buckling behavior of stiffened conical shells under axial compression were studied by Spagnoli and Chryssanthopoulos [7].

Lam and Hua [8] published a paper on rotating orthotropic conical shell vibrations which included the effects of initial hoop tension and centrifugal and Coriolis accelerations caused by rotation. They also conducted parametric studies on the frequency characteristics at different orthotropic parameters, geometric properties and rotating speeds. Spagnoli [9] studied buckling modes of axially stiffened conical shells under compressive load using the linear finite element method (LFEM). Effects of fiber directions of composite conical shells on their vibration frequencies were analyzed by $\mathrm{Hu}$ and $\mathrm{Ou}[10]$. Wu and Lee [11] studied the vibration behavior of composite conical shells with variable thickness and used firstorder shear deformation shell theory to account for the effects of transverse shear deformations. Their analysis was also applicable for cylindrical shells and circular sheets. Considering the Green strain tensor, $\mathrm{Hu}$ et al. [12] studied vibrations of twisted composite conical shells. Goldfeld 


\section{Nomenclature}

\begin{tabular}{|ll|}
\hline$A B$ & Lamé parameters \\
$k$ & Total thickness of the shell \\
$M_{\alpha}, M_{\beta}, M_{\alpha \beta}$ & Layer number \\
$N_{\alpha}, N_{\beta}, N_{\alpha \beta}$ & Nending and twisting moment resultants \\
$N$ & Total number of layers \\
$Q_{i j}^{(k)}$ & Stiffness parameters \\
$R_{\alpha}, R_{\beta}$ & Radii of curvature \\
$q_{x} q_{\theta}, q_{z}$ & Body forces \\
$u, v, w$ & Displacements at a point in the $\alpha, \beta$ and $z$ directions \\
$u_{0}, v_{0}, w_{0}$ & Displacements at the midsurface of $\alpha, \beta$ and $z$ directions \\
$\alpha, \beta$ & In-plane coordinates of the shell \\
$z$ & Out of plane coordinate of shell and distance from the middle surface \\
$\varepsilon_{\alpha}, \varepsilon_{\beta}, \gamma_{\alpha \beta}$ & Normal and in-plane shear strain \\
$\varepsilon_{0 \alpha}, \varepsilon_{0 \beta}, \gamma_{0 \alpha \beta}$ & Midsurface normal and shear strain \\
$\theta, \varphi$ & Coordinates used for shells of revolution \\
$\kappa_{\alpha}, \kappa_{\beta}$ & Curvature changes \\
$\tau$ & Changes in twist \\
$\rho$ & Density per unit volume \\
$\sigma_{\alpha}, \sigma_{\beta}, \sigma_{\alpha \beta}$ & Normal and shear stress \\
$\psi_{\alpha}, \psi_{\beta}$ & Midsurface rotations \\
$A_{i j}, B_{i j}, D_{i j}$ & Stretching, coupling and bending stiffness parameter \\
$\omega$ & Natural frequency \\
$\omega_{c}$ & Frequency parameter \\
$\mu$ & Mass per unit length \\
$E_{\alpha}, E_{\theta}$ & Young's modulus in the $\alpha, \theta$ directions \\
$L$ & Length of shell \\
$\nu_{\alpha \theta}$ & Poisson's ratio \\
$I$ & Shear modulus angle \\
$G_{\alpha \theta}$ & \\
$\varphi$ & \\
$\phi(\alpha)$ & Beam function \\
\hline
\end{tabular}

et al. [13] presented an optimal layering plan for the buckling of composite conical shells. Ross et al. [14] studied the plastic buckling of ring-stiffened conical shells under external hydrostatic pressure. Using DSC method, Civalek [15] studied vibrations of rotating conical shells. Gupta et al. [16] investigated buckling of thin conical shells under axial loads. Their study was based on laboratory tests and numerical analysis. Free vibrations of the symmetric cross-ply laminated composite conical-plate shell were investigated in [17] through transfer matrix method.

Tripathu et al. [18] studied the vibrations of composite conical shells with random material properties; they combined a finite element method with first-order perturbation technique to obtain the response statistics of the structure and found that fundamental mean natural frequency of vibration of laminated composite conical shells with all edges simply supported was at more degree for higher circumferential length to thickness $(L C / h)$ ratio. They also showed that the influence of standard deviation of fundamental natural frequency could demonstrate different sensitivity to different stacking sequences. They concluded that it is necessary to consider the effect of different lamination schemes on dispersion in frequency in designing. Civalek [19] studied vibrations of composite conical shells and used Love's first approximation thin shell theory and solved it using discrete singular convolution (DSC) method. He found that the convergence of DSC approach was very good and the results agreed well with those obtained by other researchers. His analysis such as the one presented in reference [11] is applicable for cylindrical shells and circular sheets.

Sofiyev et al. [20] studied the buckling of the FGM conical shells under hydrostatic pressure. Their parametric studies were carried out to study the influences of small radius-to-thickness ratio, lengths-to- small radius ratio and material composition on the dimensionless hydrostatic pressure of hybrid truncated conical shells. Their results revealed that the volume fraction distributions had a significant effect on the buckling pressure of FGM hybrid truncated conical shells. Sofiyev et al. [21] also studied freely supported non-homogeneous orthotropic conical shells subjected to different uniform pressures. They also extended their previous work to FGM conical shells [22]. Sofiyev [23] studied vibrations and buckling of heterogeneous orthotropic conical shells with clamped boundary conditions under external pressure. The effect of primary defect on nonlinear buckling response of FGM conical shells was investigated in reference [24]. Lachut [25] studied elastic-plastic buckling of thick steel conical shells under a combination of axial compression loads and external lateral pressure. Also, information about the non- 
linear buckling behavior of FGM conical shells subjected to axial loads was provided by Sofiyev [26], who investigated the non-linear buckling behavior of truncated conical shells made of functionally graded materials (FGMs), subject to a uniform axial compressive load, using the large deformation theory with the von Karman-Donnelltype of kinematic non-linearity. He studied the influences of the compositional profile variations and the variation of the shell geometry on the upper and lower critical axial loads. Sofiyev and Kuruoglu [27] studied non-linear buckling of composite cross-ply conical shells. Recently, Nedelcu [28] studied buckling behavior of isotropic conical shell in the linear regime based on generalized beam theory (GBT).

On the other hand, using composites, due to their special properties, has opened new horizons for researchers and industries. High mechanical, chemical and physical properties have vanished the limits of using metals and polymers. Therefore, applications of composite materials are growing day by day. Since 1980, a new type of materials called fiber metal laminates (FMLs) has been introduced [29]. These materials were produced by embedding the thin metal layers in the traditional polymerbased laminates. FMLs can improve the impact strength of the traditional composite laminates. This type of composite technology was originally developed at the University of Delft in Netherlands. Embedded aluminum sheets in the traditional layers were alternatively stuck together. Arall was the first FML type which was a mixture of aluminum sheets with Aramide epoxy composite layers. In 1980, the Glare composite was introduced which was used in aircraft industry as the heat resistance to airflow. Some researchers showed that this type of composite had better resistance against fatigue and impact loading compared with the traditionally composite laminates. In fact, FML has the average properties of the metal and polymerbased composite materials. Light weight and fatigue resistance are other features of the FMLs. The unique properties of conical shells with broad applications in major industries such as aviation reveals the necessity of study on their mechanical behavior.

Hence, in this paper the vibrational behavior of the FML conical shells is predicted. In this regard, thin shell assumptions are used to find fundamental equations and Hamilton's principle is applied to derive governing equations. So far, numerical techniques have been used for solving the governing equations of conical shells. In the present work, the Galerkin analytical method is used to solve them. Also, in most previous papers only simply supported boundary conditions have been considered, whereas boundary conditions have a significant effect on the vibrational response of the structure. In the present study, an analogy is established between conical shells and beams, and beam functions are used to approximate the field variables in the axial direction. So a good approximation of conical shells under various boundary conditions can be obtained through the use of this technique.

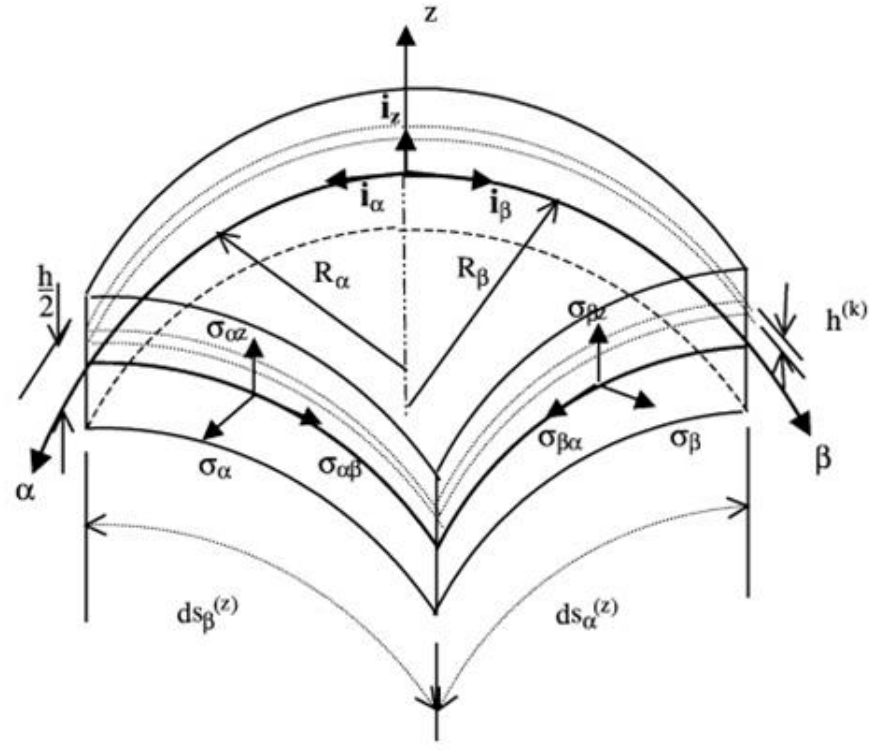

Fig. 1. Geometry and coordinate systems for a truncated conical shell.

\section{Preliminaries}

\subsection{Geometric relations}

Considering the symbols used in Figure 1 for a shell, the strains can be considered as follows [30]:

$$
\begin{aligned}
\varepsilon_{\alpha} & =\varepsilon_{0 \alpha}+z k_{\alpha} \\
\varepsilon_{\beta} & =\varepsilon_{0 \beta}+z k_{\beta} \\
\gamma_{\alpha \beta} & =\gamma_{0 \alpha \beta}+z \tau
\end{aligned}
$$

where $\varepsilon_{0 \alpha}, \varepsilon_{0 \beta}$ are the mid-plane strains in the meridional and circumferential directions and $\gamma_{0 \alpha \beta}$ is the midplane shear strain in $\alpha-\beta$ plane. $k_{\alpha}, k_{\beta}$ are the curvature changes and $\tau$ is the change in twist. The linear straindisplacement relations and associated changes for thin shells are:

$$
\begin{aligned}
\varepsilon_{0 \alpha} & =\frac{1}{A} \frac{\partial u_{0}}{\partial \alpha}+\frac{v_{0}}{A B} \frac{\partial A}{\partial \beta}+\frac{w_{0}}{R_{\alpha}} \\
\varepsilon_{0 \beta} & =\frac{1}{B} \frac{\partial v_{0}}{\partial \beta}+\frac{u_{0}}{A B} \frac{\partial B}{\partial \alpha}+\frac{w_{0}}{R_{\beta}} \\
\gamma_{0 \alpha \beta} & =\frac{1}{A} \frac{\partial v_{0}}{\partial \alpha}-\frac{u_{0}}{A B} \frac{\partial A}{\partial \beta}+\frac{1}{B} \frac{\partial u_{0}}{\partial \beta}-\frac{v_{0}}{A B} \frac{\partial B}{\partial \alpha}+2 \frac{w_{0}}{R_{\alpha \beta}} \\
k_{\alpha} & =\frac{1}{A} \frac{\partial \psi_{\alpha}}{\partial \alpha}+\frac{\psi_{\beta}}{A B} \frac{\partial A}{\partial B}, \quad k_{\beta}=\frac{1}{B} \frac{\partial \psi_{\beta}}{\partial \beta}+\frac{\psi_{\alpha}}{A B} \frac{\partial B}{\partial \alpha} \\
\tau & =\frac{1}{A} \frac{\partial \psi_{\beta}}{\partial \alpha}-\frac{\psi_{\alpha}}{A B} \frac{\partial A}{\partial \beta}+\frac{1}{B} \frac{\partial \psi_{\alpha}}{\partial \beta}-\frac{\psi_{\beta}}{A B} \frac{\partial B}{\partial \alpha}
\end{aligned}
$$

where

$$
\psi_{\alpha}=\frac{u_{0}}{R_{\alpha}}+\frac{v_{0}}{R_{\alpha \beta}}-\frac{1}{A} \frac{\partial w_{0}}{\partial \alpha}, \quad \psi_{\beta}=\frac{v_{0}}{R_{\beta}}+\frac{u_{0}}{R_{\alpha \beta}}-\frac{1}{A} \frac{\partial w_{0}}{\partial \beta}
$$


In which $u_{0}, v_{0}$ and $w_{0}$ are middle surface displacements of the shell and $A, B$ are parameters of Lamé. Also, $R_{\alpha}$ and $R_{\beta}$ are radii of curvatures $\alpha, \beta$ and $R_{\alpha \beta}$ is the radius of twist, respectively.

\subsection{Constitutive equations}

By applying the hypothesis of Kirchhoff based on eliminating the shear deformations and neglecting the $\varepsilon_{z}$, the stress-strain relations for an element of the material in the $k$ th layer are defined as:

$$
\left[\begin{array}{c}
\sigma_{\alpha} \\
\sigma_{\beta} \\
\sigma_{\alpha \beta}
\end{array}\right]_{k}=\left[\begin{array}{lll}
Q_{11} & Q_{12} & Q_{16} \\
Q_{12} & Q_{22} & Q_{26} \\
Q_{16} & Q_{26} & Q_{66}
\end{array}\right]_{k}\left[\begin{array}{c}
\varepsilon_{\alpha} \\
\varepsilon_{\beta} \\
\gamma_{\alpha \beta}
\end{array}\right]_{k}
$$

where $\sigma_{\alpha}$ and $\sigma_{\beta}$ are normal stresses in $\alpha$ and $\beta$ direction, respectively and $\sigma_{\alpha \beta}$ is the in-plane shear stress component. Additionally, $\varepsilon_{\alpha}$ and $\varepsilon_{\beta}$ are normal strains and $\gamma_{\alpha \beta}$ is the in-plane engineering shear strain, and $Q_{i j}$ represents the elements of reduced stiffness matrix for the FML structure. If the shell plane $(\alpha \beta)$ becomes parallel or perpendicular to the fibers, both the $Q_{16}$ and $Q_{26}$ become zero.

By integrating the stresses along the shell thickness $(h)$, the resultant forces and moments can be obtained as

$$
\begin{aligned}
& {\left[\begin{array}{c}
N_{\alpha} \\
N_{\beta} \\
N_{\alpha \beta}
\end{array}\right]=\int_{-h / 2}^{h / 2}\left[\begin{array}{c}
\sigma_{\alpha} \\
\sigma_{\beta} \\
\sigma_{\alpha \beta}
\end{array}\right] \mathrm{d} z} \\
& {\left[\begin{array}{c}
M_{\alpha} \\
M_{\beta} \\
M_{\alpha \beta}
\end{array}\right]=\int_{-h / 2}^{h / 2}\left[\begin{array}{c}
\sigma_{\alpha} \\
\sigma_{\beta} \\
\sigma_{\alpha \beta}
\end{array}\right] z \mathrm{~d} z}
\end{aligned}
$$

By substituting equations (1)-(6) into equations (7) and (8), afterwards, by layer to layer integration along the shell thickness, one finds that

$$
\left[\begin{array}{c}
N_{\alpha} \\
N_{\beta} \\
N_{\alpha \beta} \\
M_{\alpha} \\
M_{\beta} \\
M_{\alpha \beta}
\end{array}\right]=\left[\begin{array}{cccccc}
A_{11} & A_{12} & A_{16} & B_{11} & B_{12} & B_{16} \\
A_{12} & A_{22} & A_{26} & B_{12} & B_{22} & B_{26} \\
A_{16} & A_{26} & A_{66} & B_{16} & B_{26} & B_{66} \\
B_{11} & B_{12} & B_{16} & D_{11} & D_{12} & D_{16} \\
B_{12} & B_{22} & B_{26} & D_{12} & D_{22} & D_{26} \\
B_{16} & B_{26} & B_{66} & D_{16} & D_{26} & D_{66}
\end{array}\right]\left[\begin{array}{c}
\varepsilon_{0 \alpha} \\
\varepsilon_{0 \beta} \\
\gamma_{0 \alpha \beta} \\
k_{\alpha} \\
k_{\beta} \\
\tau
\end{array}\right]
$$

in which $A_{i j}, B_{i j}$ and $D_{i j}$ are stiffness coefficients which could be obtained in the following form

$$
\begin{aligned}
& A_{i j}=\sum_{k=1}^{N} Q_{i j}^{(k)}\left(z_{k}-z_{k-1}\right) \\
& B_{i j}=\frac{1}{2} \sum_{k=1}^{N} Q_{i j}^{(k)}\left(z_{k}^{2}-z_{k-1}^{2}\right) \\
& D_{i j}=\frac{1}{3} \sum_{k=1}^{N} Q_{i j}^{(k)}\left(z_{k}^{3}-z_{k-1}^{3}\right)
\end{aligned}
$$

where $z_{k}$ is the distance of the middle surface of the shell from the middle surface of the $k$ th layer. It should be noted that, for the composite shells which are symmetric with respect to their middle surface, $B_{i j}=0$.

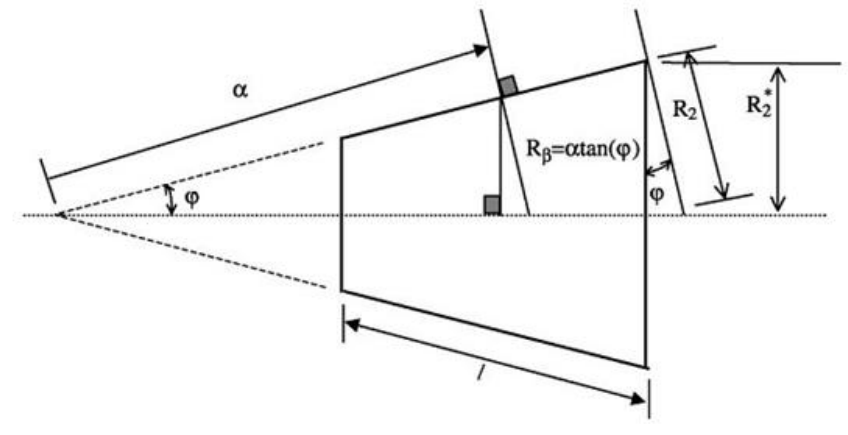

Fig. 2. Side view of a truncated conical shell.

\section{Governing equations}

\subsection{Shell geometry}

Figure 2 shows the side view of a truncated conical shell. The shell-form equation can be written as follows

$$
(\mathrm{d} s)^{2}=(\mathrm{d} \alpha)^{2}+\alpha^{2} \sin ^{2}(\varphi)(\mathrm{d} \theta)^{2}
$$

where $\mathrm{d} s$ is the differential of the arc length measured along the surface and $\theta$ denotes the circumferential coordinate. Using equation (11), Lamé parameters and the radius of curvature values are obtained as:

$$
\begin{aligned}
A & =1, \quad B=\alpha \sin (\varphi) \\
R_{\alpha} & =\infty, \quad R_{\beta}=\alpha \tan (\varphi)
\end{aligned}
$$

\subsection{Kinematics relations}

By substituting equation (12) into equations (2)-(4), middle surfaces trains curvature changes and changes in twist for thin truncated conical shells can be rewritten as:

$$
\begin{aligned}
\varepsilon_{0 \alpha} & =\frac{\partial u_{0}}{\partial \alpha} \\
\varepsilon_{0 \theta} & =\frac{1}{\alpha \sin (\varphi)} \frac{\partial v_{0}}{\partial \theta}+\frac{u_{0}}{\alpha}+\frac{w_{0}}{\alpha \tan (\varphi)} \\
\gamma_{0 \alpha \theta} & =\frac{\partial v_{0}}{\partial \alpha}+\frac{1}{\alpha \sin (\varphi)} \frac{\partial u_{0}}{\partial \theta}-\frac{v_{0}}{\alpha} \\
k_{\alpha} & =\frac{\partial \psi_{\alpha}}{\partial \alpha} \\
k_{\theta} & =\frac{1}{\alpha \sin (\varphi)} \frac{\partial \psi_{\theta}}{\partial \theta}+\frac{\psi_{\alpha}}{\alpha} \\
\tau & =\frac{\partial \psi_{\theta}}{\partial \alpha}+\frac{1}{\alpha \sin (\varphi)} \frac{\partial \psi_{\alpha}}{\partial \theta}-\frac{\psi_{\theta}}{\alpha}
\end{aligned}
$$

where $\psi_{\alpha}=-\frac{\partial w_{0}}{\partial \alpha}, \quad \psi_{\alpha}=\frac{v_{0}}{\alpha \tan (\varphi)}-\frac{1}{\alpha \sin (\varphi)} \frac{\partial w_{0}}{\partial \theta}$ according to equation (5). 
Table 1. Values of $\alpha_{i}, \lambda_{m}$ and $\varsigma_{m}$ for different boundary conditions [32].

\begin{tabular}{|c|c|c|c|}
\hline Boundary conditions & $\alpha_{i}(i=1,2,3,4)$ & $\lambda_{m}$ & $\zeta_{m}$ \\
\hline simply-simply (S-S) & $\alpha_{1}=0, \alpha_{2}=0$ & $m \pi$ & 1 \\
\hline$\Delta 80080000008$ & $\alpha_{3}=0, \alpha_{4}=-1$ & & \\
\hline clamped-clamped (C-C) & $\alpha_{1}=1, \alpha_{2}=-1$ & $\cos \lambda_{m} \cosh \lambda_{m}=1$ & $\frac{\cosh \lambda_{m}-\cos \lambda_{m}}{\sinh \lambda_{m}-\sin \lambda_{m}}$ \\
\hline & \multicolumn{3}{|l|}{$\alpha_{3}=1, \alpha_{4}=-1$} \\
\hline \multirow{2}{*}{ free-free $(\mathrm{F}-\mathrm{F})$} & \multirow{2}{*}{$\alpha_{1}=1, \alpha_{2}=1$} & \multirow{2}{*}{$\cos \lambda_{m} \cosh \lambda_{m}=1$} & $\underline{\cosh \lambda_{m}-\cos \lambda_{m}}$ \\
\hline & & & $\sinh \lambda_{m}-\sin \lambda_{m}$ \\
\hline \multirow{3}{*}{ clamped-simply (C-S) } & \multirow[b]{2}{*}{$\alpha_{1}=1, \alpha_{2}=-1$} & & $\cosh \lambda_{m}-\cos \lambda_{m}$ \\
\hline & & $\tan \lambda_{m}=\tanh \lambda_{m}$ & $\frac{\sinh \lambda_{m}-\sin \lambda_{m}}{\sin }$ \\
\hline & $\alpha_{3}=1, \alpha_{4}=-1$ & & \\
\hline \multirow{2}{*}{ clamped-free (C-F) } & \multirow{2}{*}{$\alpha_{1}=1, \alpha_{2}=-1$} & \multirow{2}{*}{$\cos \lambda_{m} \cosh \lambda_{m}=-1$} & $\sinh \lambda_{m}-\sin \lambda_{m}$ \\
\hline & & & $\overline{\cosh \lambda_{m}+\cos \lambda_{m}}$ \\
\hline & $\alpha_{3}=1, \alpha_{4}=-1$ & & \\
\hline \multirow{2}{*}{ free-simply (F-S) } & \multirow{2}{*}{$\alpha_{1}=1, \alpha_{2}=1$} & \multirow{2}{*}{$\tan \lambda_{m}=\tanh \lambda_{m}$} & $\cosh \lambda_{m}-\cos \lambda_{m}$ \\
\hline & & & $\overline{\sinh \lambda_{m}-\sin \lambda_{m}}$ \\
\hline 500000000000 & $\alpha_{3}=1, \alpha_{4}=1$ & & \\
\hline
\end{tabular}

\subsection{Motion equations}

Using the Hamilton's principle, the equations of motion for the conical shells can be obtained as:

$$
\begin{array}{r}
\frac{\partial N_{\alpha}}{\partial x}+\frac{N_{\alpha}-N_{\theta}}{\alpha}+\frac{1}{\alpha \sin (\varphi)} \frac{\partial N_{\alpha \theta}}{\partial \theta}+q_{x}=I \ddot{u}_{0}^{2} \\
\frac{1}{\alpha \sin (\varphi)} \frac{\partial N_{\theta}}{\partial \theta}+\frac{\partial N_{\alpha \theta}}{\partial \alpha}+\frac{2 N_{\alpha \theta}}{\alpha}+\frac{Q_{\theta}}{\alpha \tan (\varphi)}+q_{\theta}=I \ddot{v}_{0}^{2} \\
-\left(\frac{N_{\theta}}{\alpha \tan (\varphi)}\right)+\frac{\partial Q_{\alpha}}{\partial \alpha}+\frac{Q_{\alpha}}{\alpha}+\frac{1}{\alpha \sin (\varphi)} \frac{\partial Q_{\theta}}{\partial \theta}+q_{z}=I \ddot{w}_{0}^{2}
\end{array}
$$

where $Q_{\alpha}=\frac{M_{\alpha}}{\alpha}+\frac{\partial M_{\alpha}}{\partial \alpha}+\frac{1}{\alpha \sin (\varphi)} \frac{\partial M_{\theta \alpha}}{\partial \theta}-\frac{M_{\theta}}{\alpha}$ and $Q_{\theta}=$ $\frac{1}{\alpha \sin (\varphi)} \frac{\partial M_{\theta}}{\partial \theta}+\frac{\partial M_{\alpha \theta}}{\partial \alpha}+\frac{2 M_{\alpha \theta}}{\alpha}$, and $I$ denotes moment of inertia which is defined as $I=\sum_{k=1}^{N} \int_{h_{k-1}}^{h_{k}} \rho^{(k)} \mathrm{d} z$ where $\rho^{(k)}$ is the density of $k$ th layer.

Equations (9) and (13) are substituted into equation (14) and the equations of motion can be obtained in terms of displacements as follows:

$$
\begin{array}{r}
{\left[\begin{array}{lll}
L_{11} & L_{12} & L_{13} \\
L_{21} & L_{22} & L_{23} \\
L_{31} & L_{32} & L_{33}
\end{array}\right]\left[\begin{array}{l}
u_{0} \\
v_{0} \\
w_{0}
\end{array}\right]+\left[\begin{array}{ccc}
-I & 0 & 0 \\
0 & -I & 0 \\
0 & 0 & -I
\end{array}\right]} \\
= \\
=\left[\begin{array}{l}
-q_{\alpha} \\
-q_{\beta} \\
-q_{z}
\end{array}\right]
\end{array}
$$

where the coefficients $L_{i j}$ are introduced in the appendix. These complicated equations can be greatly simplified if the multi-layer conical shells are considered symmetric.

\section{Beam functions}

Beam functions are one of the most common choices for field variables of rectangular plates and also cylindrical and conical shells. To this end, beam and shell boundary conditions should be the same, which is based on the principle that the behavior of an axial strip of the conical shell should be similar to the beam behavior with the similar boundary conditions [31]. Applying beam functions makes it possible to analyze the vibration of the conical shell in various boundary conditions. In order to find desired beam functions, the equation of motion for the beam is described as:

$$
\frac{\partial^{2}}{\partial \alpha^{2}}\left(E I \frac{\partial^{2} \phi}{\partial \alpha^{2}}\right)=-\mu \frac{\partial^{2} \phi}{\partial t^{2}}+q(\alpha)
$$

where $E$ and $\mu$ are Young's modulus and the mass per unit length of the beam, respectively, $\phi(\alpha)$ is the transverse displacement at position $\alpha$, and $q(\alpha)$ is the external load. By solving the above equation, the following function can be obtained [32]

$$
\begin{aligned}
\phi(\alpha)= & \alpha_{1} \cosh \left(\frac{\lambda_{m} \alpha}{L}\right)+\alpha_{2} \cos \left(\frac{\lambda_{m} \alpha}{L}\right) \\
& -\varsigma_{m}\left(\alpha_{3} \sinh \left(\frac{\lambda_{m} \alpha}{L}\right)+\alpha_{4} \sin \left(\frac{\lambda_{m} \alpha}{L}\right)\right)
\end{aligned}
$$

where $\alpha_{i}(i=1,2, \ldots, 4)$ are the constants that can be -1 , 0 or 1 according to the boundary conditions. $\lambda_{m}$ denotes transcendental equation roots obtained from boundary conditions and $\varsigma_{m}$ is a parameter related to $\lambda_{m}$. The values of $\alpha_{i}, \lambda_{m}$ and $\varsigma_{m}$ are tabulated in Table 1 for different boundary conditions. The mathematical form of the geometric boundary conditions of the clamped, simply supported and free boundary conditions can be expressed as follows:

- Clamped (C) boundary condition

$$
\phi(\alpha)=\phi^{\prime}(\alpha)=0
$$


- Free (F) boundary condition

$$
\phi^{\prime \prime}(\alpha)=\phi^{\prime \prime \prime}(\alpha)=0
$$

- Simply supported (S) boundary condition

$$
\phi(\alpha)=\phi^{\prime \prime}(\alpha)=0
$$

\section{Vibrational analysis using the Galerkin method}

In the absence of external loads $\left(q_{\alpha}=q_{\beta}=q_{z}=0\right)$ and considering that each point of the elastic system experiences a harmonic motion in every natural frequency, the displacement field can be written as [31]:

$$
\begin{aligned}
u_{0}(\alpha, \theta, t) & =A u(\alpha, \theta) \mathrm{e}^{j \omega t}=A \frac{\partial \phi(\alpha)}{\partial \alpha} \cos (n \theta) \mathrm{e}^{j \omega t} \\
v_{0}(\alpha, \theta, t) & =B v(\alpha, \theta) \mathrm{e}^{j \omega t}=B \phi(\alpha) \sin (n \theta) \mathrm{e}^{j \omega t} \\
w_{0}(\alpha, \theta, t) & =C w(\alpha, \theta) \mathrm{e}^{j \omega t}=C \phi(\alpha) \cos (n \theta) \mathrm{e}^{j \omega t}
\end{aligned}
$$

where $\omega$ denotes the natural frequency, $n$ is the circumferential wave number, and $A, B$ and $C$ are the constant coefficients. Moreover, three functions of $u(\alpha, \theta), v(\alpha, \theta)$ and $w(\alpha, \theta)$ form a natural mode. By embedding equation (21) in equation (15), the following relations can be obtained

$$
\begin{aligned}
& L_{1}\left\{u_{0}, v_{0}, w_{0}\right\}+A \rho h \omega^{2} u \mathrm{e}^{j \omega t}=0 \\
& L_{2}\left\{u_{0}, v_{0}, w_{0}\right\}+B \rho h \omega^{2} v \mathrm{e}^{j \omega t}=0 \\
& L_{3}\left\{u_{0}, v_{0}, w_{0}\right\}+C \rho h \omega^{2} w \mathrm{e}^{j \omega t}=0
\end{aligned}
$$

where $L_{1}\left\{u_{0}, v_{0}, w_{0}\right\}=L_{11} u_{0}+L_{12} v_{0}+L_{13} w_{0}$ similarly $L_{2}\left\{u_{0}, v_{0}, w_{0}\right\}=L_{21} u_{0}+L_{22} v_{0}+L_{23} w_{0}$ and $L_{3}\left\{u_{0}, v_{0}, w_{0}\right\}=L_{31} u_{0}+L_{32} v_{0}+L_{33} w_{0}$.

The Galerkin method is implemented herein. To this end, the first equation of equation (22) is multiplied by $u$, the second equation by $v$ and the third one by $w$, as weight functions. Afterwards, integrating them over the domain results in

$$
\begin{aligned}
& \int_{\alpha} \int_{\theta}\left[L_{1}\left\{u_{0}, v_{0}, w_{0}\right\}+A \rho h \omega^{2} u \mathrm{e}^{j \omega t}\right] u \alpha \sin \varphi \mathrm{d} \theta \mathrm{d} \alpha=0 \\
& \int_{\alpha} \int_{\theta}\left[L_{2}\left\{u_{0}, v_{0}, w_{0}\right\}+B \rho h \omega^{2} v \mathrm{e}^{j \omega t}\right] v \alpha \sin \varphi \mathrm{d} \theta \mathrm{d} \alpha=0 \\
& \int_{\alpha} \int_{\theta}\left[L_{3}\left\{u_{0}, v_{0}, w_{0}\right\}+C \rho h \omega^{2} w \mathrm{e}^{j \omega t}\right] w \alpha \sin \varphi \mathrm{d} \theta \mathrm{d} \alpha=0
\end{aligned}
$$

By performing the integration, the homogeneous system of equations will be in the following form

$$
\left[\begin{array}{ccc}
\rho h \omega^{2}+k_{11} & k_{12} & k_{13} \\
k_{21} & \rho h \omega^{2}+k_{22} & k_{23} \\
k_{31} & k_{32} & \rho h \omega^{2}+k_{33}
\end{array}\right]\left\{\begin{array}{l}
A \\
B \\
C
\end{array}\right\}=0
$$

in which

$$
\begin{aligned}
k_{11} & =\frac{\int_{\alpha} \int_{\theta} L_{11}\{u\} u \alpha \sin \varphi \mathrm{d} \theta \mathrm{d} \alpha}{\int_{\alpha} \int_{\theta} u^{2} \alpha \sin \varphi \mathrm{d} \theta \mathrm{d} \alpha} \\
k_{12} & =\frac{\int_{\alpha} \int_{\theta} L_{12}\{v\} u \alpha \sin \varphi \mathrm{d} \theta \mathrm{d} \alpha}{\int_{\alpha} \int_{\theta} u^{2} \alpha \sin \varphi \mathrm{d} \theta \mathrm{d} \alpha} \\
k_{33} & =\frac{\int_{\alpha} \int_{\theta} L_{33}\{w\} w \alpha \sin \varphi \mathrm{d} \theta \mathrm{d} \alpha}{\int_{\alpha} \int_{\theta} w^{2} \alpha \sin \varphi \mathrm{d} \theta \mathrm{d} \alpha}
\end{aligned}
$$

To get a non-trivial solution, the determinant of the coefficient matrix must be equal to zero. Thus, the characteristic equation of the coefficient matrix of equation (24) is as follows:

$$
\omega^{6}+a_{1} \omega^{4}+a_{2} \omega^{2}+a_{3}=0
$$

where the coefficients $a_{i}(i=1,2,3)$ are given by

$$
\begin{gathered}
a_{1}=\frac{1}{\rho h}\left(k_{11}+k_{22}+k_{33}\right) \\
a_{2}=\frac{1}{(\rho h)^{2}}\left(k_{11} k_{22}+k_{11} k_{33}+k_{22} k_{33}-k_{23}^{2}-k_{12}^{2}-k_{13}^{2}\right) \\
a_{3}=-\frac{1}{(\rho h)^{3}}\left(k_{11} k_{23}^{2}-k_{11} k_{22} k_{33}+k_{22} k_{13}^{2}+k_{33} k_{12}^{2}\right. \\
\left.-2 k_{12} k_{23} k_{13}\right)
\end{gathered}
$$

By solving the algebraic equation (28) the following three natural frequencies of the shell could be achieved

$$
\begin{aligned}
& \omega_{1}^{2}=-\frac{2}{3} \sqrt{a_{1}^{2}-3 a_{2}} \cos \frac{\lambda}{3}-\frac{a_{1}}{3} \\
& \omega_{2}^{2}=-\frac{2}{3} \sqrt{a_{1}^{2}-3 a_{2}} \cos \frac{\lambda+2 \pi}{3}-\frac{a_{1}}{3} \\
& \omega_{3}^{2}=-\frac{2}{3} \sqrt{a_{1}^{2}-3 a_{2}} \cos \frac{\lambda+4 \pi}{3}-\frac{a_{1}}{3}
\end{aligned}
$$

in which

$$
\lambda=\cos ^{-1} \frac{27 a_{3}+2 a_{1}^{3}-9 a_{1} a_{2}}{2 \sqrt{\left(a_{1}^{2}-3 a_{2}\right)^{3}}}
$$

The non-dimensional parameter that could be used in the results is defined as follows:

$$
\text { Frequency parameter } \omega_{c}=\sqrt{\frac{\rho h}{A_{11}}} \omega R_{2}^{*}
$$

where $\omega$ is the lowest natural frequency.

\section{Results and discussions}

\subsection{Verification}

To validate the results obtained by the present approach, the frequency parameter of an isotropic conical 
Table 2. Comparison of frequency parameters for the isotropic conical shell [33,34].

\begin{tabular}{cccccccccc}
\hline \multirow{2}{*}{$n$} & \multicolumn{9}{c}{$\alpha=30^{\circ}$} \\
\cline { 2 - 9 } & {$[33]$} & {$[34]$} & Present work & {$[33]$} & {$[34]$} & Present work & {$[33]$} & {$[34]$} & Present work \\
\hline 2 & 0.7910 & 0.8431 & 0.8405 & 0.6879 & 0.7642 & 0.7639 & 0.5722 & 0.6342 & 0.6342 \\
3 & 0.7284 & 0.7416 & 0.7375 & 0.6973 & 0.7211 & 0.7204 & 0.6001 & 0.6236 & 0.6235 \\
4 & 0.6352 & 0.6419 & 0.6368 & 0.6664 & 0.6747 & 0.6737 & 0.6054 & 0.6146 & 0.6144 \\
5 & 0.5531 & 0.5590 & 0.5536 & 0.6304 & 0.6336 & 0.6325 & 0.6077 & 0.6113 & 0.6111 \\
6 & 0.4949 & 0.5008 & 0.4955 & 0.6032 & 0.6049 & 0.6037 & 0.6159 & 0.6172 & 0.6170 \\
7 & 0.4653 & 0.4701 & 0.4661 & 0.5918 & 0.5928 & 0.5919 & 0.6343 & 0.6347 & 0.6346 \\
8 & 0.4645 & 0.4687 & 0.4653 & 0.5992 & 0.6005 & 0.5994 & 0.6650 & 0.6653 & 0.6651 \\
\hline
\end{tabular}

Table 3. Comparison of frequency parameter for the composite conical shell [35].

\begin{tabular}{ccc}
\hline$h / R_{2}^{*}$ & {$[35]$} & Present work \\
\hline 0.01 & 0.1799 & 0.1671 \\
0.02 & 0.2153 & 0.2088 \\
0.03 & 0.2397 & 0.2174 \\
0.04 & 0.2620 & 0.2480 \\
0.05 & 0.2841 & 0.2705 \\
0.06 & 0.3061 & 0.2981 \\
0.07 & 0.3277 & 0.3265 \\
0.08 & 0.3484 & 0.3477 \\
0.09 & 0.3680 & 0.3669 \\
0.10 & 0.3863 & 0.3845 \\
\hline
\end{tabular}

shell taken from references $[33,34]$ is considered in Table 2. Some geometrical and mechanical properties of the shell are as follows

$$
h=0.004 \mathrm{~m}, \quad \frac{h}{R_{2}^{*}}=0.01, \quad \frac{L \sin (\varphi)}{R_{2}^{*}}=0.25, \quad \nu=0.3
$$

Table 2 presents the frequency parameter (Eq. (36)) for the first axial mode of a conical shell with S-S boundary conditions, different circumferential half-waves and also for different half-angles of the conical shells. The results show that the frequency parameters calculated by the Galerkin method are in good agreement with those from other references [33,34].

To further investigate the validation of the current method, the vibrational response of the conical composites shell obtained by the present work and by reference [35] are tabulated in Table 3. Shell characteristics are assumed as follows:

$$
\begin{aligned}
\frac{L \sin (\varphi)}{R_{2}^{*}} & =0.5, \quad \varphi=30^{\circ}, \quad \frac{E_{\alpha}}{E_{\theta}}=15.0, \\
\nu_{\alpha \theta} & =0.25, \quad \frac{G_{\alpha \theta}}{E_{\theta}}=0.5
\end{aligned}
$$

In this table, there are the values of frequency parameters of non-symmetric cross-ply conical composite shell with 2 layers $[0,90]$ and the boundary conditions $(\mathrm{S}-\mathrm{S})$ for different thicknesses. The results of this table confirm that the presented method can strongly analyze the vibration of conical composite shell.
Table 4. Effects of positioning of the aluminum layers on natural frequency of the conical FML shell (Boundary conditions: C-S).

\begin{tabular}{cccccc}
\hline & \multicolumn{5}{c}{ Position of aluminum layer } \\
\cline { 2 - 6 }$n$ & $(1,10)$ & $(2,9)$ & $(3,8)$ & $(4,7)$ & $(5,6)$ \\
\hline 1 & 0.6904 & 0.6905 & 0.6904 & 0.6905 & 0.6904 \\
3 & 0.3205 & 0.3201 & 0.3201 & 0.3199 & 0.3199 \\
5 & 0.1974 & 0.1949 & 0.1953 & 0.1939 & 0.1941 \\
7 & 0.1643 & 0.1549 & 0.1567 & 0.1523 & 0.1529 \\
9 & 0.1892 & 0.1727 & 0.1766 & 0.1679 & 0.1692 \\
\hline
\end{tabular}

\subsection{Characteristics of FML shells}

To study the FML conical shells in more detail, a tenlayer shell with some Aramid reinforced plastics (ARP) layers and with the following characteristics is selected, $h=0.0002 \mathrm{~m}, G=1.8 \mathrm{GPa}, E_{12}=95 \mathrm{GPa}, E_{13}=$ $5.1 \mathrm{GPa}, \nu=0.34, \rho=1320 \mathrm{~kg} . \mathrm{cm}^{-3}$.

Also, some aluminum layers are selected with the following characteristics, $h=0.0002 \mathrm{~m}, G=28 \mathrm{GPa}$, $E=72.4 \mathrm{GPa}, \nu=0.33, \rho=2780 \mathrm{~kg} . \mathrm{cm}^{-3}$.

In this study, four common boundary conditions of shell(S-S, C-C, C-S, C-F) are investigated and the transverse half-wave is considered to be $m=1$.

The geometrical parameters of the conical shell are $L=0.5 \mathrm{~m}, R_{1}=0.25 \mathrm{~m}$, its phase angle is $\theta=\frac{\pi}{7} \mathrm{rad}$ and its conical semi-vertex angle is $\alpha=\frac{\pi}{12} \mathrm{rad}$.

\subsection{Effect of aluminum layers}

To investigate the effect of aluminum layers, they are symmetrically put in some layers of the laminate. In other words, if an aluminum layer is put in the 1st layer of the laminate, simultaneously it is put in the 10th layer too. This is also done for the layers $(2,9),(3,8),(4,7)$ and $(5,6)$. For each mentioned cases, frequency parameter is calculated in wave numbers of $n=1$ to 9 . It should be noted that the lowest frequency parameter is reached in $n=7$, where if the system is enforced the same external frequency, the resonance will happen and the system may be damaged. Therefore, in this study, instead of using $n=1$, the wave number $n=7$ is utilized to produce the lowest frequency of the system, as can be seen from Table 4 .

The effect of increasing the aluminum layers on the system frequency parameter for different boundary 


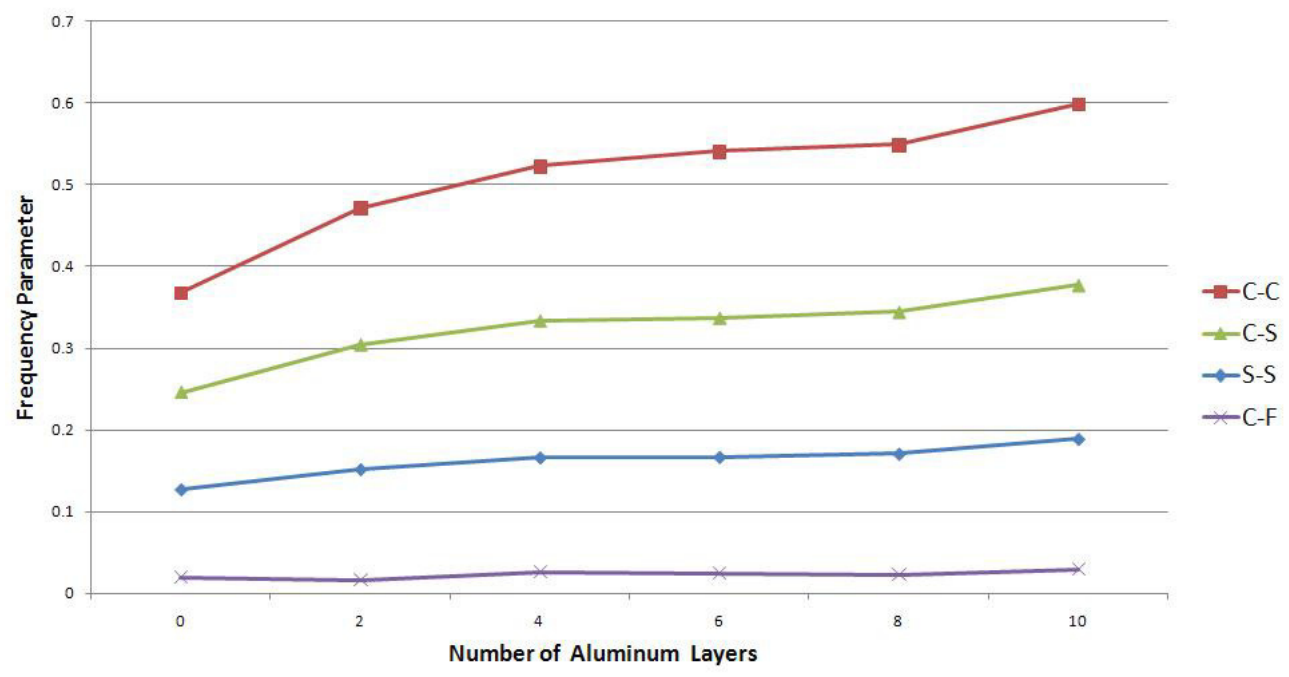

Fig. 3. The effect of increasing the aluminum layers on the system frequency parameter for different boundary conditions.

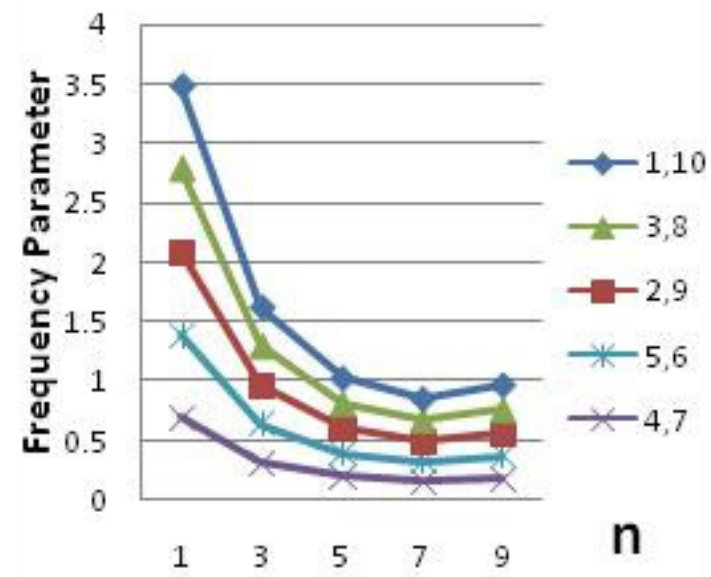

Fig. 4. The effect of embedding two aluminum layers on the system frequency parameter (boundary conditions: C-C).

conditions is depicted in Figure 3. One can be observed from this figure that the frequency parameter has a sudden decrease in $\mathrm{C}-\mathrm{F}$ boundary conditions, at point 2, which indicated the presence of two aluminum layers. This treatment happens due to selecting the smallest natural frequency in different lay ups of the structure, meaning that, in selection of natural frequency, several cases can be gained if the aluminum in layer is put in layers $(9$, 2 ) or in layers $(10,1)$ or etc. Figure 3 also shows that by increasing the number of the aluminum layers in the structure, the frequency parameter increases too. It can be concluded that the C-S, S-S and C-C boundary conditions have nearly no effect on increasing the frequency parameter. Moreover, no matter where and how many aluminum layers are embedded in the structure, there will be a significant increase in the frequency parameter of the system.

The effects of embedding two aluminum layers in the frequency parameter of the system are presented in Figures 4-7 for different boundary conditions. No matter

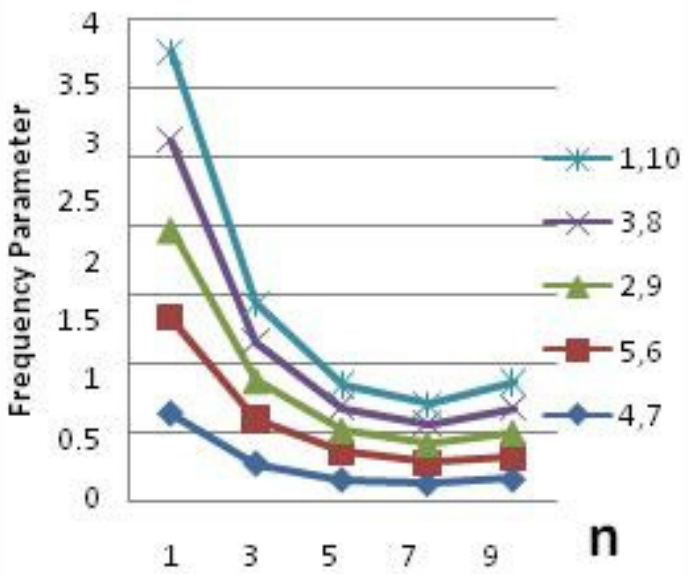

Fig. 5. The effect of embedding two aluminum layers on the system frequency parameter (boundary conditions: S-S).

what the boundary conditions are, if a couple of aluminum layers is embedded in a truncated conical FML shell, the best places are in the 1st and 10th layers (i.e. in the first and last layers), which leads to the highest frequency of the system in most of the frequency numbers. In other ones, if this value does not become higher than others, it will be very close to them.

\subsection{Effect of cone length}

In this subsection, the effect of cone length on frequency parameter of the FML shell is studied. The results are generated when aluminum layers are embedded in 1st, 2nd, 9th and 10th layers of the shell, the boundary conditions are supposed to be C-F and C-C, and four aluminums are placed from 10 composite layers. The results are presented graphically in Figures 8 and 9.

It is observed from Figures 8 and 9 that an increase in cone length of the FML shell results in the reduction 


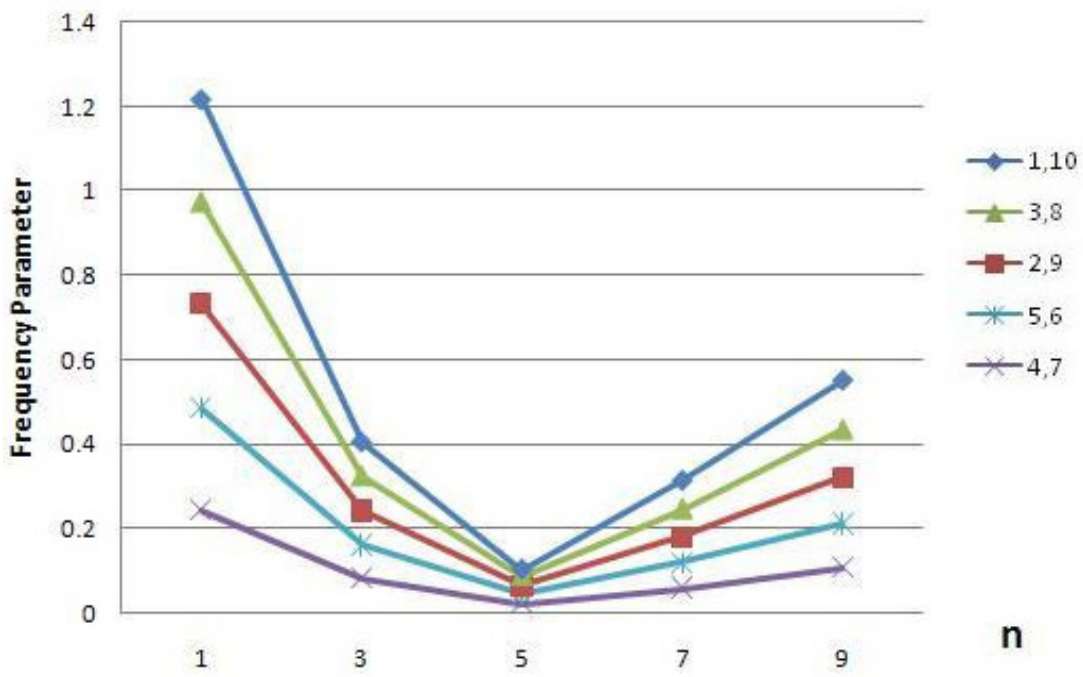

Fig. 6. The effect of embedding two aluminum layers on the system frequency parameter (boundary conditions: C-F).

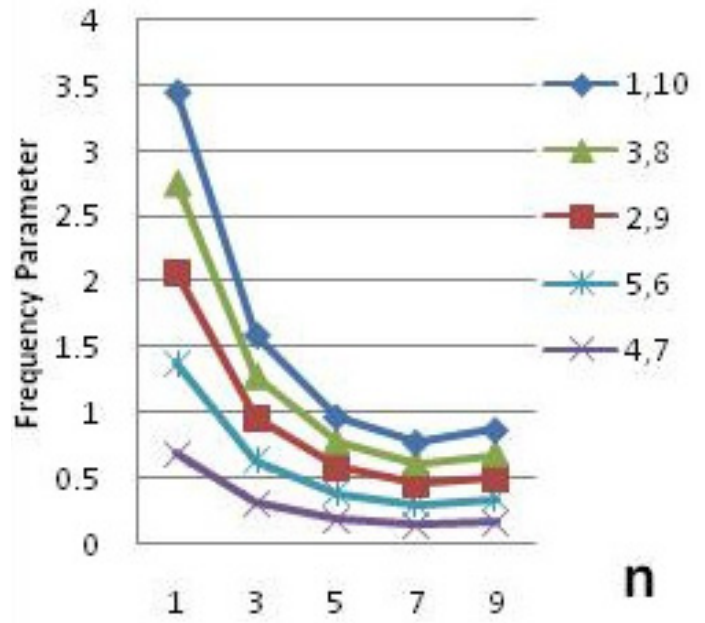

Fig. 7. The effect of embedding two aluminum layers on the system frequency parameter (boundary conditions: C-S).

of the system frequency parameter. Therefore, if the system is enforced by an external frequency, it experiences the resonance. However the amount of this frequency is greater in the first frequency number $(n=1)$ which should be considered in design processes. Also, the system frequency parameters are greater for C-C boundary conditions in comparison to the $\mathrm{C}-\mathrm{F}$ ones. This change is more severe for $n=1$ and nearly mode rate for $n>1$.

\subsection{Effect of cone angle}

\subsubsection{C-F boundary conditions}

Figure 10 indicates the effect of cone vertex half-angle on the system frequency parameter for C-F boundary conditions when the aluminum layers are put in the 1st, 2nd, 9 th and 10th layers of the structure. If $R_{2}$ is kept constant, increasing the radius $R_{1}$ will result in an increase in half-apex angle of the cone (see Fig. 2), and the system frequency parameter increases for $n=1-5$. However, for wave numbers $(n)$ greater than 5 , this becomes the other way around. Obviously, due to the large effect of resonance for lower frequency numbers (e.g. for $n=1$ ), the effect of this phenomenon becomes less.

\subsubsection{C-C Boundary conditions}

Figure 11 shows the effect of decreasing the conical half-vertex angle (or increasing of the $R_{1}$ ) of the conical FML shell on the system frequency parameter for C-C boundary conditions. In this type of boundary conditions, increase of the radius of the smaller circle of the cone or decrease of the cone half-apex angle results in an increase in frequency parameter of the FML shells for all the frequency numbers.

\subsection{Effect of boundary conditions}

For a 10 layered conical FML shell, 31 cases can exist by embedding the aluminum layers in different places of the structure (from 2 layers up to 10 ones). It must be noted that all the above cases could be investigated in 4 different types of boundary conditions as C-C, S-S, C-S, C-F.

To facilitate the evaluation of results, arrangement of all different lay ups of the FML structure is briefly presented in Table 5. For each of the 31 different lay ups, numbers 1 to 31 are used, because of the place of aluminum layers in the structure. For example, code 2 means that aluminum layers are put in the 1st and 10th layers of the structure, etc.

The effect of using aluminum layers on the system frequency parameter of the conical FML shells is shown in Figure 12, which indicates that an increase in using the aluminum layers in FML conical shells results in an 


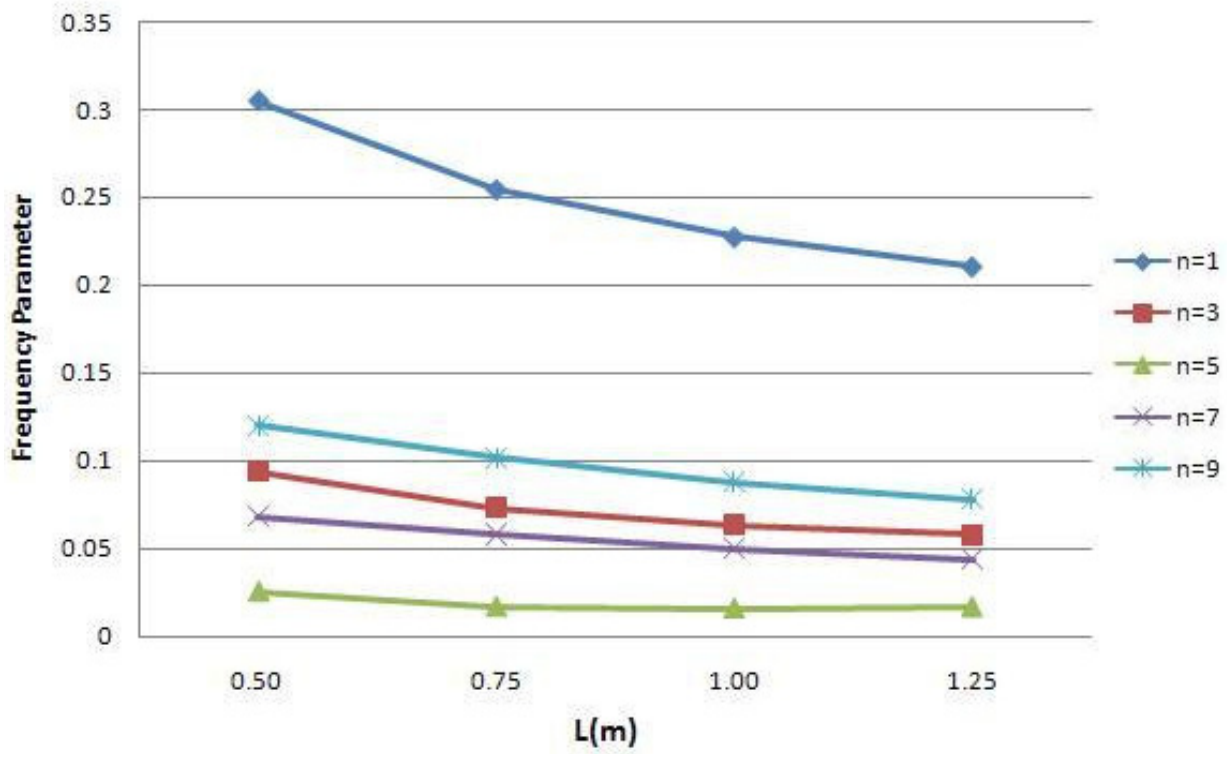

Fig. 8. Effect of cone length on the system frequency parameter (boundary conditions: C-F).

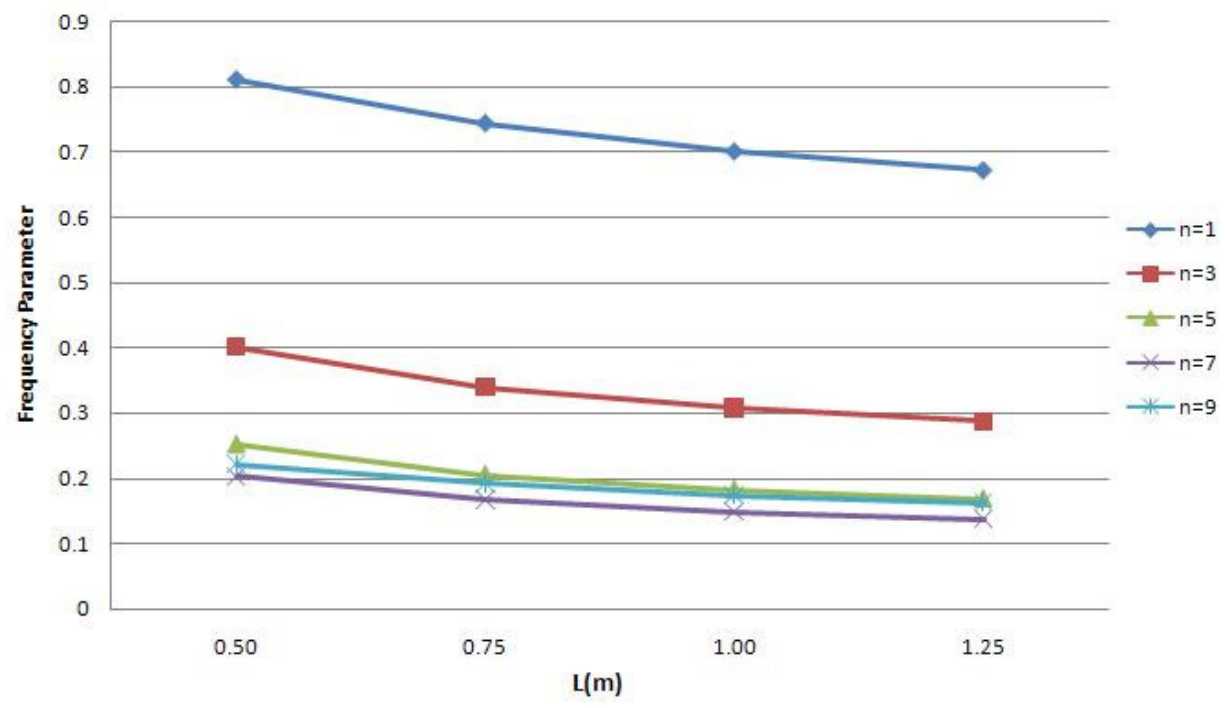

Fig. 9. Effect of cone length on the system frequency parameter (boundary conditions: C-C).

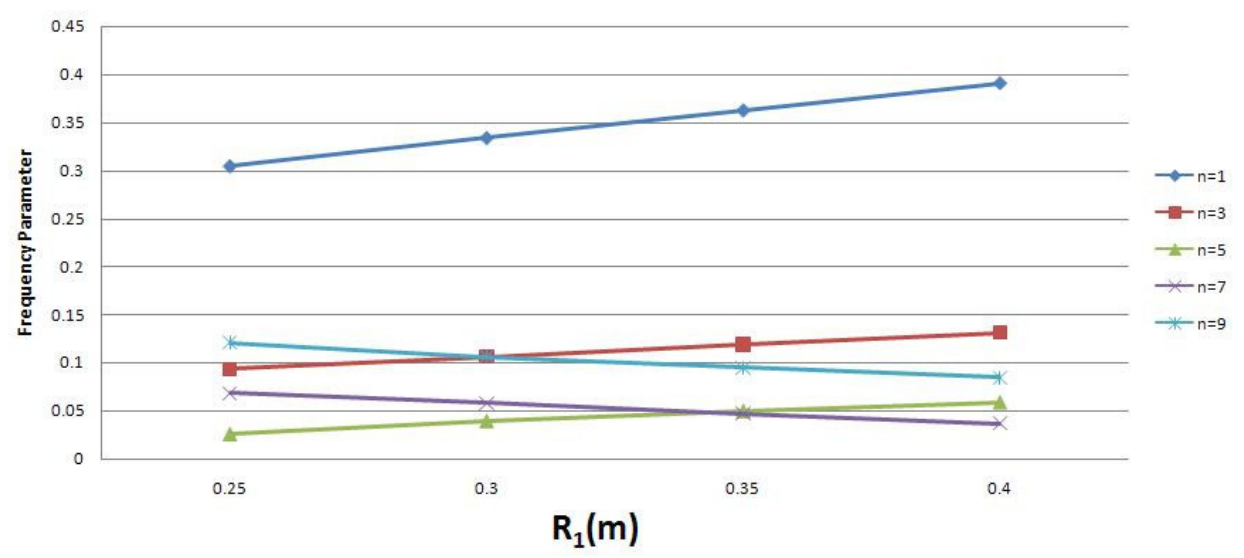

Fig. 10. The effect of cone vertex half-angle on the system frequency parameter (boundary conditions: C-F). 


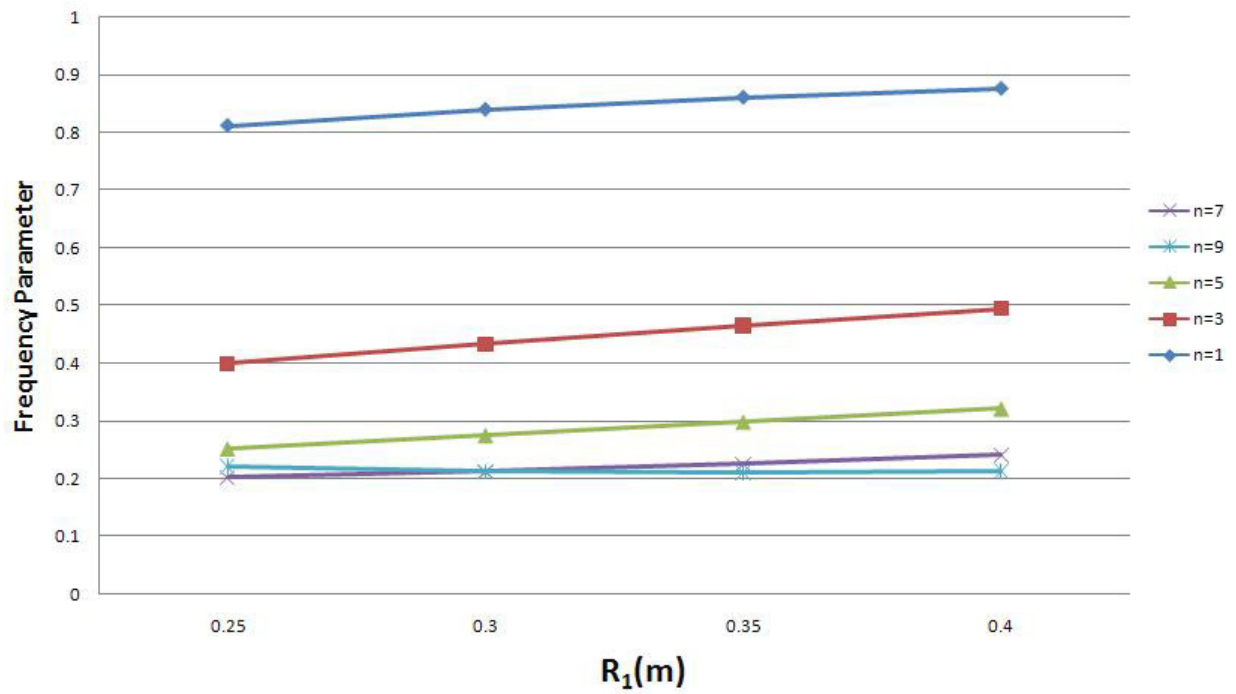

Fig. 11. The effect of cone vertex half-angle on the system frequency parameter (boundary conditions: C-C).

Table 5. Code numbers of 31 different lay ups of the FML shell, due to the place of the aluminum layers in the structure.

\begin{tabular}{|c|c|c|c|}
\hline Codes & $\begin{array}{l}\text { Location of embedding } \\
\text { aluminum layers }\end{array}$ & Codes & $\begin{array}{c}\text { Location of embedding } \\
\text { aluminum layers }\end{array}$ \\
\hline 1 & 0 & 17 & $1,2,3,8,9,10$ \\
\hline 2 & 1,10 & 18 & $1,2,4,7,9,10$ \\
\hline 3 & 2,9 & 19 & $1,2,5,6,9,10$ \\
\hline 4 & 3,8 & 20 & $1,3,4,7,8,10$ \\
\hline 5 & 4,7 & 21 & $1,3,5,6,9,10$ \\
\hline 6 & 5,6 & 22 & $1,4,5,6,7,10$ \\
\hline 7 & $1,2,9,10$ & 23 & $2,3,4,7,8,9$ \\
\hline 8 & $1,3,8,10$ & 24 & $2,3,5,6,8,9$ \\
\hline 9 & $1,4,7,10$ & 25 & $2,4,5,6,7,9$ \\
\hline 10 & $1,5,6,10$ & 26 & $1,2,3,4,7,8,9,10$ \\
\hline 11 & $2,3,8,9$ & 27 & $1,2,3,5,6,8,9,10$ \\
\hline 12 & $2,4,7,9$ & 28 & $1,2,4,5,6,7,9,10$ \\
\hline 13 & $2,5,6,9$ & 29 & $1,3,4,5,6,7,8,10$ \\
\hline 14 & $3,4,7,8$ & 30 & $2,3,4,5,6,7,8,9$ \\
\hline 15 & $3,5,6,8$ & 31 & $1,2,3,4,5,6,7,8,9,10$ \\
\hline 16 & $4,5,6,7$ & & \\
\hline
\end{tabular}

increase in system frequency parameter. However, there is one disagreement in the figure for the layer code 21 (Tab. 5), which may be due to the use of beam functions for solving motion equations of the shell. In higher system frequency numbers, this point yields an increase of system frequency parameter. As can be observed, effects of using aluminum in FML shells are more obvious in $n=1$. Also, as mentioned before, this frequency number should receive more notice. The same behavior can be almost seen for other boundary conditions.

As stated earlier, the effects of boundary conditions are important, so in order to further investigations, the effect of different 31 lay ups of conical FML shells is studied here. Considering Figure 13, the effect of S-S, C-S, $\mathrm{C}-\mathrm{C}$ boundary conditions on the frequency parameter of FML shell is almost the same. However, the values that belonged to the C-F boundary conditions are very different from other boundary conditions. Figure 13 also shows that a free (F) boundary condition on one side of the conical FML shell strongly reduces the frequency parameter. This undesired result decreases the usage of these structures in industry.

\section{Conclusion}

This paper presented the possibility of covering some defects of composite materials (e.g. improving the vibrational characteristics of the conical shells) by combining them with some metals such as aluminum layers. According to this approach: embedding the aluminum layers in the traditional composite laminate shells (here, in truncated conical ones) showed a good effect by increasing the frequency parameter of the structure. However, in C-F boundary conditions, lower values were obtained for the system frequency parameter, which indicated that using 


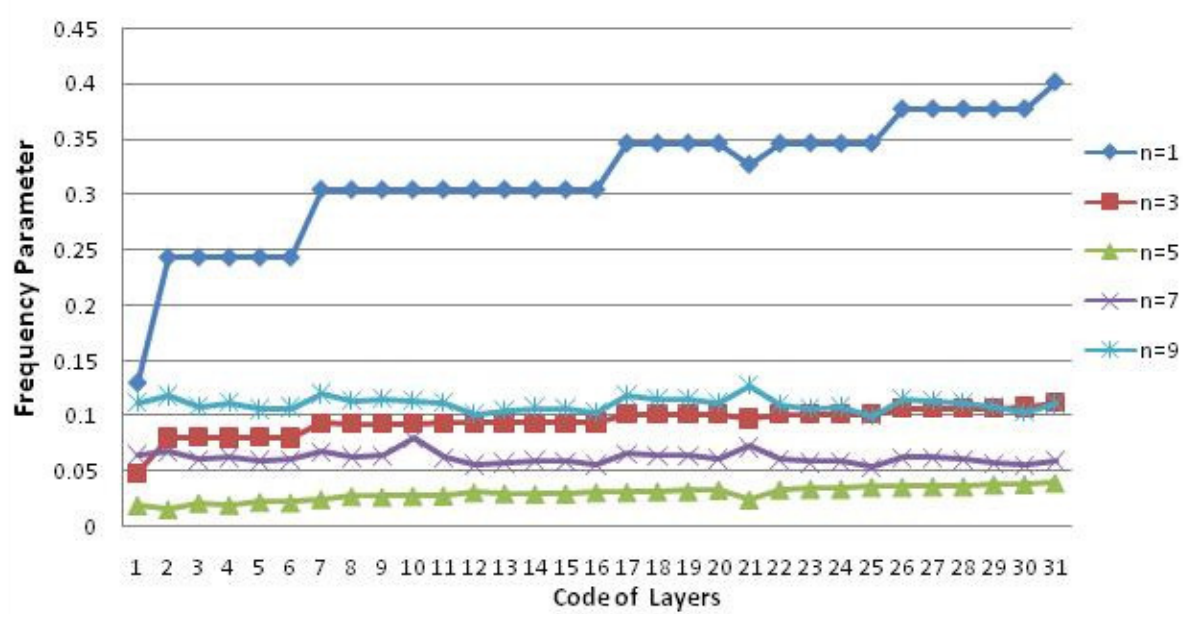

Fig. 12. The effect of lay up of aluminum layers on the system frequency parameters (boundary conditions: C-F).

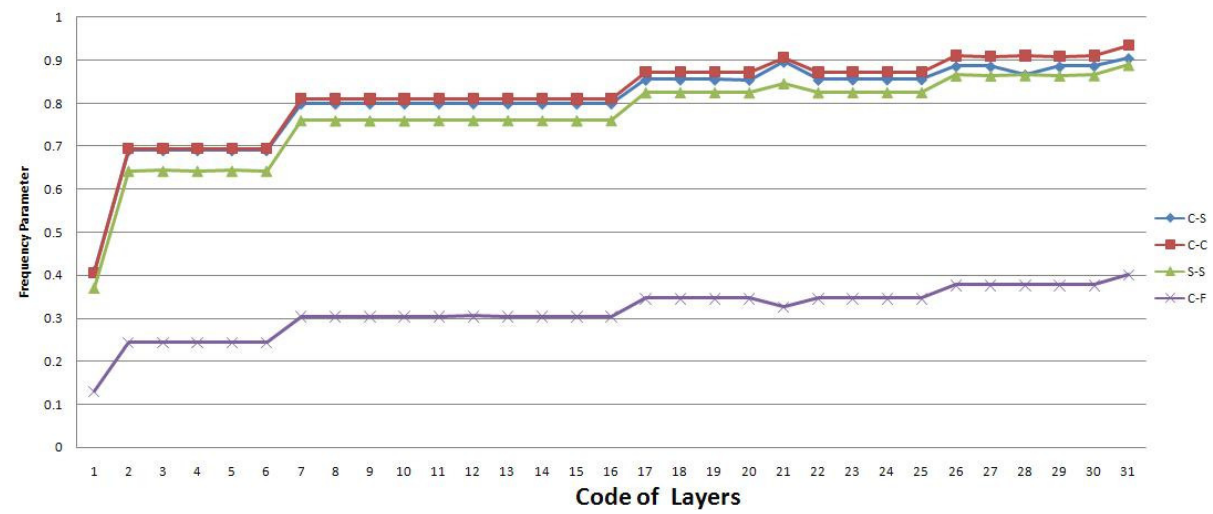

Fig. 13. The effect of boundary conditions on system frequency parameter of the conical FML shell for $n=1$.

the aluminum layers cannot improve the vibrational characteristics of the structure for this kind of boundary conditions. For C-C boundary conditions, the best result (the highest improvement in system frequency parameter) was obtained.

- According to the results, when only a couple of aluminum layers were embedded in a conical FML shell symmetrically, the best place to embed the aluminum layers was in the 1st and 10th layers. This type of layering led to the highest natural frequency in all frequency counters and in all boundary conditions.

- It was observed that, in S-S boundary conditions, an increase in thickness of the structure increased the system frequency parameter. However, this mostly occurred in smaller frequency numbers; meaning that smaller frequency numbers were more important than other ones.

- It was shown that, for C-C and C-F boundary conditions, an increase in the length of conical FML shells resulted in the decrease in frequency parameter of the shell. Moreover, in C-C boundary conditions, frequency parameters were higher than $\mathrm{C}-\mathrm{F}$ ones.

- This research also demonstrated that an increase in $R_{1}$ or a decrease in half-vertex cone caused an increase in the natural frequency of the conical FML shells. By increasing $R_{1}$, the shell tended to become a cylinder. Therefore, the conical shells were more sensitive to the resonance compared with the cylindrical ones.

- It was also indicated that the more the aluminum layers are embedded in the structure, the more the structure frequency parameter increases.

\section{Appendix}

The defined coefficients $L_{i j}$ in equation (15) can be expressed as [30]:

$$
\begin{aligned}
L_{11}= & \frac{\partial}{\partial \alpha}\left(A_{11}\left(\frac{\partial}{\partial \alpha}\right)+A_{12}\left(\frac{1}{\alpha}\right)+A_{16}\left(\frac{1}{\alpha \sin (\varphi)} \frac{\partial}{\partial \theta}\right)\right) \\
& +\frac{1}{\alpha}\left(A_{11}\left(\frac{\partial}{\partial \alpha}\right)+A_{12}\left(\frac{1}{\alpha}\right)+A_{16}\left(\frac{1}{\alpha \sin (\varphi)} \frac{\partial}{\partial \theta}\right)\right) \\
& -\frac{1}{\alpha}\left(A_{12}\left(\frac{\partial}{\partial \alpha}\right)+A_{22}\left(\frac{1}{\alpha}\right)+A_{26}\left(\frac{1}{\alpha \sin (\varphi)} \frac{\partial}{\partial \theta}\right)\right) \\
& +\frac{1}{\alpha \sin (\varphi)} \frac{\partial}{\partial \theta}\left(A_{16}\left(\frac{\partial}{\partial \alpha}\right)+A_{26}\left(\frac{1}{\alpha}\right)\right. \\
& \left.+A_{66}\left(\frac{1}{\alpha \sin (\varphi)} \frac{\partial}{\partial \theta}\right)\right)
\end{aligned}
$$




$$
\begin{aligned}
L_{12}= & \frac{\partial}{\partial \alpha}\left(A_{12}\left(\frac{1}{\alpha \sin (\varphi)} \frac{\partial}{\partial \theta}\right)+A_{16}\left(\frac{\partial}{\partial \alpha}-\frac{1}{\alpha}\right)+B_{12}\left(\frac{\cot (\varphi)}{\alpha^{2} \sin (\varphi)} \frac{\partial}{\partial \theta}\right)+B_{16}\left(\frac{\cot (\varphi)}{\alpha} \frac{\partial}{\partial \alpha}-\frac{2 \cot (\varphi)}{\alpha^{2}}\right)\right) \\
& +\frac{1}{\alpha}\left(A_{12}\left(\frac{1}{\alpha \sin (\varphi)} \frac{\partial}{\partial \theta}\right)+A_{16}\left(\frac{\partial}{\partial \alpha}-\frac{1}{\alpha}\right)+B_{12}\left(\frac{\cot (\varphi)}{\alpha^{2} \sin (\varphi)} \frac{\partial}{\partial \theta}\right)+B_{16}\left(\frac{\cot (\varphi)}{\alpha} \frac{\partial}{\partial \alpha}-\frac{2 \cot (\varphi)}{\alpha^{2}}\right)\right) \\
& -\frac{1}{\alpha}\left(A_{22}\left(\frac{1}{\alpha \sin (\varphi)} \frac{\partial}{\partial \theta}\right)+A_{26}\left(\frac{\partial}{\partial \alpha}-\frac{1}{\alpha}\right)+B_{22}\left(\frac{\cot (\varphi)}{\alpha^{2} \sin (\varphi)} \frac{\partial}{\partial \theta}\right)+B_{26}\left(\frac{\cot (\varphi)}{\alpha} \frac{\partial}{\partial \alpha}-\frac{2 \cot (\varphi)}{\alpha^{2}}\right)\right) \\
& +\frac{1}{\alpha \sin (\varphi)} \frac{\partial}{\partial \theta}\left(A_{26}\left(\frac{1}{\alpha \sin (\varphi)} \frac{\partial}{\partial \theta}\right)+A_{66}\left(\frac{\partial}{\partial \alpha}-\frac{1}{\alpha}\right)+B_{26}\left(\frac{\cot (\varphi)}{\alpha^{2} \sin (\varphi)} \frac{\partial}{\partial \theta}\right)+B_{66}\left(\frac{\cot (\varphi)}{\alpha} \frac{\partial}{\partial \alpha}-\frac{2 \cot (\varphi)}{\alpha^{2}}\right)\right)
\end{aligned}
$$

$$
\begin{aligned}
L_{13}= & \frac{\partial}{\partial \alpha}\left(A_{12}\left(\frac{1}{\alpha \tan (\varphi)}\right)-B_{11}\left(\frac{\partial^{2}}{\partial \alpha^{2}}\right)-B_{12}\left(\frac{1}{\alpha^{2} \sin ^{2}(\varphi)} \frac{\partial^{2}}{\partial \theta^{2}}+\frac{1}{\alpha} \frac{\partial}{\partial \alpha}\right)\right. \\
& \left.+B_{16}\left(-\frac{2}{\alpha \sin (\varphi)} \frac{\partial^{2}}{\partial \theta \partial \alpha}+\frac{2}{\alpha^{2} \sin (\varphi)} \frac{\partial}{\partial \theta}\right)\right)+\frac{1}{\alpha}\left(A_{12}\left(\frac{1}{\alpha \tan (\varphi)}\right)-B_{11}\left(\frac{\partial^{2}}{\partial \alpha^{2}}\right)\right. \\
& \left.-B_{12}\left(\frac{1}{\alpha^{2} \sin ^{2}(\varphi)} \frac{\partial^{2}}{\partial \theta^{2}}+\frac{1}{\alpha} \frac{\partial}{\partial \alpha}\right)+B_{16}\left(-\frac{2}{\alpha \sin (\varphi)} \frac{\partial^{2}}{\partial \theta \partial \alpha}+\frac{2}{\alpha^{2} \sin (\varphi)} \frac{\partial}{\partial \theta}\right)\right) \\
& -\frac{1}{\alpha}\left(A_{22}\left(\frac{1}{\alpha \tan (\varphi)}\right)-B_{12}\left(\frac{\partial^{2}}{\partial \alpha^{2}}\right)-B_{22}\left(\frac{1}{\alpha^{2} \sin ^{2}(\varphi)} \frac{\partial^{2}}{\partial \theta^{2}}+\frac{1}{\alpha} \frac{\partial}{\partial \alpha}\right)\right. \\
& \left.+B_{26}\left(-\frac{2}{\alpha \sin (\varphi)} \frac{\partial^{2}}{\partial \theta \partial \alpha}+\frac{2}{\alpha^{2} \sin (\varphi)} \frac{\partial}{\partial \theta}\right)\right)+\frac{1}{\alpha \sin (\varphi)} \frac{\partial}{\partial \theta}\left(A_{26}\left(\frac{1}{\alpha \tan (\varphi)}\right)-B_{16}\left(\frac{\partial^{2}}{\partial \alpha^{2}}\right)\right. \\
& \left.-B_{26}\left(\frac{1}{\alpha^{2} \sin ^{2}(\varphi)} \frac{\partial^{2}}{\partial \theta^{2}}+\frac{1}{\alpha} \frac{\partial}{\partial \alpha}\right)+B_{66}\left(-\frac{2}{\alpha \sin (\varphi)} \frac{\partial^{2}}{\partial \theta \partial \alpha}+\frac{2}{\alpha^{2} \sin (\varphi)} \frac{\partial}{\partial \theta}\right)\right)
\end{aligned}
$$

$$
\begin{aligned}
L_{21}= & \frac{1}{\alpha \sin (\varphi)} \frac{\partial}{\partial \theta}\left(A_{12}\left(\frac{\partial}{\partial \alpha}\right)+A_{22}\left(\frac{1}{\alpha}\right)+A_{26}\left(\frac{1}{\alpha \sin (\varphi)} \frac{\partial}{\partial \theta}\right)\right)+\frac{\partial}{\partial \alpha}\left(A_{16}\left(\frac{\partial}{\partial \alpha}\right)+A_{26}\left(\frac{1}{\alpha}\right)\right. \\
& \left.+A_{66}\left(\frac{1}{\alpha \sin (\varphi)} \frac{\partial}{\partial \theta}\right)\right)+\frac{2}{\alpha}\left(A_{16}\left(\frac{\partial}{\partial \alpha}\right)+A_{26}\left(\frac{1}{\alpha}\right)+A_{66}\left(\frac{1}{\alpha \sin (\varphi)} \frac{\partial}{\partial \theta}\right)\right)+\frac{\cos (\varphi)}{\alpha^{2} \sin ^{2}(\varphi)} \frac{\partial}{\partial \theta}\left(B_{12}\left(\frac{1}{\partial \alpha}\right)\right. \\
& \left.+B_{22}\left(\frac{\mu_{0}}{\alpha}\right)+B_{26}\left(\frac{1}{\alpha \sin (\varphi)} \frac{\partial}{\partial \theta}\right)\right)+\frac{1}{\alpha \tan (\varphi)} \frac{\partial}{\partial \alpha}\left(B_{16}\left(\frac{\partial}{\partial \alpha}\right)+B_{26}\left(\frac{1}{\alpha}\right)\right. \\
& \left.+B_{66}\left(\frac{1}{\alpha \sin (\varphi)} \frac{\partial}{\partial \theta}\right)\right)+\frac{2}{\alpha^{2} \tan (\varphi)}\left(B_{16}\left(\frac{\partial}{\partial \alpha}\right)+B_{26}\left(\frac{1}{\alpha}\right)+B_{66}\left(\frac{1}{\alpha \sin (\varphi)} \frac{\partial}{\partial \theta}\right)\right)
\end{aligned}
$$

$$
\begin{aligned}
L_{22}= & \frac{1}{\alpha \sin (\varphi)} \frac{\partial}{\partial \theta}\left(A_{22}\left(\frac{1}{\alpha \sin (\varphi)} \frac{\partial}{\partial \theta}\right)+A_{26}\left(\frac{\partial}{\partial \alpha}-\frac{1}{\alpha}\right)+B_{22}\left(\frac{\cos (\varphi)}{\alpha^{2} \sin ^{2}(\varphi)} \frac{\partial}{\partial \theta}\right)+B_{26}\left(\frac{\cot (\varphi)}{\alpha} \frac{\partial}{\partial \alpha}-\frac{2 \cot (\varphi)}{\alpha^{2}}\right)\right) \\
& +\frac{\partial}{\partial \alpha}\left(A_{26}\left(\frac{1}{\alpha \sin (\varphi)} \frac{\partial}{\partial \theta}\right)+A_{66}\left(\frac{\partial}{\partial \alpha}-\frac{1}{\alpha}\right)+B_{26}\left(\frac{\cot (\varphi)}{\alpha^{2} \sin ^{2}(\varphi)} \frac{\partial}{\partial \theta}\right)+B_{66}\left(\frac{\cot (\varphi)}{\alpha} \frac{\partial}{\partial \alpha}-\frac{2 \cot (\varphi)}{\alpha^{2}}\right)\right) \\
& +\frac{2}{\alpha}\left(A_{26}\left(\frac{1}{\alpha \sin (\varphi)} \frac{\partial}{\partial \theta}\right)+A_{66}\left(\frac{\partial}{\partial \alpha}-\frac{1}{\alpha}\right)+B_{26}\left(\frac{\cot (\varphi)}{\alpha^{2} \sin ^{2}(\varphi)} \frac{\partial}{\partial \theta}\right)+B_{66}\left(\frac{\cot (\varphi)}{\alpha} \frac{\partial}{\partial \alpha}-\frac{2 \cot (\varphi)}{\alpha^{2}}\right)\right) \\
& +\frac{\cos (\varphi)}{\alpha^{2} \sin ^{2}(\varphi)} \frac{\partial}{\partial \theta}\left(B_{22}\left(\frac{1}{\alpha \sin (\varphi)} \frac{\partial}{\partial \theta}\right)+B_{26}\left(\frac{\partial}{\partial \alpha}-\frac{1}{\alpha}\right)+D_{22}\left(\frac{\cot (\varphi)}{\alpha^{2} \sin ^{2}(\varphi)} \frac{\partial}{\partial \theta}\right)\right) \\
& +D_{26}\left(\frac{\cot (\varphi)}{\alpha} \frac{\partial}{\partial \alpha}-\frac{2 \cot (\varphi)}{\alpha^{2}}\right) \frac{1}{\alpha \tan (\varphi)} \frac{\partial}{\partial \alpha}\left(B_{26}\left(\frac{1}{\alpha \sin (\varphi)} \frac{\partial}{\partial \theta}\right)+B_{66}\left(\frac{\partial}{\partial \alpha}-\frac{1}{\alpha}\right)\right. \\
& \left.+D_{26}\left(\frac{\cot (\varphi)}{\alpha^{2} \sin ^{2}(\varphi)} \frac{\partial}{\partial \theta}\right)+D_{66}\left(\frac{\cot (\varphi)}{\alpha} \frac{\partial}{\partial \alpha}-\frac{2 \cot (\varphi)}{\alpha^{2}}\right)\right)+\frac{2}{\alpha^{2} \tan (\varphi)}\left(B_{26}\left(\frac{1}{\alpha \sin (\varphi)} \frac{\partial}{\partial \theta}\right)\right. \\
& \left.+B_{66}\left(\frac{\partial}{\partial \alpha}-\frac{1}{\alpha}\right)+D_{26}\left(\frac{\cot (\varphi)}{\alpha^{2} \sin ^{2}(\varphi)} \frac{\partial}{\partial \theta}\right)+D_{66}\left(\frac{\cot (\varphi)}{\alpha} \frac{\partial}{\partial \alpha}-\frac{2 \cot (\varphi)}{\alpha^{2}}\right)\right)
\end{aligned}
$$




$$
\begin{aligned}
L_{23}= & \frac{1}{\alpha \sin (\varphi)} \frac{\partial}{\partial \theta}\left(A_{22}\left(\frac{1}{\alpha \tan (\varphi)}\right)-B_{12}\left(\frac{\partial^{2}}{\partial \alpha^{2}}\right)-B_{22}\left(\frac{1}{\alpha^{2} \sin ^{2}(\varphi)} \frac{\partial^{2}}{\partial \theta^{2}}+\frac{1}{\alpha} \frac{\partial}{\partial \alpha}\right)\right. \\
& \left.+B_{26}\left(-\frac{2}{\alpha \sin (\varphi)} \frac{\partial^{2}}{\partial \theta \partial \alpha}+\frac{2}{\alpha^{2} \sin ^{2}(\varphi)} \frac{\partial}{\partial \theta}\right)\right)+\frac{\partial}{\partial \alpha}\left(A_{26}\left(\frac{1}{\alpha \tan (\varphi)}\right)-B_{16}\left(\frac{\partial^{2}}{\partial \alpha^{2}}\right)\right. \\
& \left.-B_{26}\left(\frac{1}{\alpha^{2} \sin ^{2}(\varphi)} \frac{\partial^{2}}{\partial \theta^{2}}+\frac{1}{\alpha} \frac{\partial}{\partial \alpha}\right)+B_{66}\left(-\frac{2}{\alpha \sin (\varphi)} \frac{\partial^{2}}{\partial \theta \partial \alpha}+\frac{2}{\alpha^{2} \sin ^{2}(\varphi)} \frac{\partial}{\partial \theta}\right)\right) \\
& +\frac{2}{\alpha}\left(A_{26}\left(\frac{1}{\alpha \tan (\varphi)}\right)-B_{16}\left(\frac{\partial^{2}}{\partial \alpha^{2}}\right)-B_{26}\left(\frac{1}{\alpha^{2} \sin ^{2}(\varphi)} \frac{\partial^{2}}{\partial \theta^{2}}+\frac{1}{\alpha} \frac{\partial}{\partial \alpha}\right)\right. \\
& \left.+B_{66}\left(-\frac{2}{\alpha \sin (\varphi)} \frac{\partial^{2}}{\partial \theta \partial \alpha}+\frac{2}{\alpha^{2} \sin ^{2}(\varphi)} \frac{\partial}{\partial \theta}\right)\right)+\frac{\cos (\varphi)}{\alpha^{2} \sin ^{2}(\varphi)} \frac{\partial}{\partial \theta}\left(B_{22}\left(\frac{1}{\alpha \tan (\varphi)}\right)-D_{12}\left(\frac{\partial^{2}}{\partial \alpha^{2}}\right)\right. \\
& \left.-D_{22}\left(\frac{1}{\alpha^{2} \sin ^{2}(\varphi)} \frac{\partial^{2}}{\partial \theta^{2}}+\frac{1}{\alpha} \frac{\partial}{\partial \alpha}\right)+D_{26}\left(-\frac{2}{\alpha \sin (\varphi)} \frac{\partial^{2}}{\partial \theta \partial \alpha}+\frac{2}{\alpha^{2} \sin ^{2}(\varphi)} \frac{\partial}{\partial \theta}\right)\right) \\
& +\frac{1}{\alpha \tan (\varphi)} \frac{\partial}{\partial \alpha}\left(B_{26}\left(\frac{1}{\alpha \tan (\varphi)}\right)-D_{16}\left(\frac{\partial^{2}}{\partial \alpha^{2}}\right)-D_{26}\left(\frac{1}{\alpha^{2} \sin ^{2}(\varphi)} \frac{\partial^{2}}{\partial \theta^{2}}+\frac{1}{\alpha} \frac{\partial}{\partial \alpha}\right)\right. \\
& \left.+D_{66}\left(-\frac{2}{\alpha \sin ^{2}(\varphi)} \frac{\partial^{2}}{\partial \theta \partial \alpha}+\frac{2}{\alpha^{2} \sin ^{2}(\varphi)} \frac{\partial}{\partial \theta}\right)\right)+\frac{2}{\alpha^{2} \tan (\varphi)}\left(B_{26}\left(\frac{1}{\alpha \tan (\varphi)}\right)-D_{16}\left(\frac{\partial^{2}}{\partial \alpha^{2}}\right)\right. \\
& \left.-D_{26}\left(\frac{1}{\alpha^{2} \sin ^{2}(\varphi)} \frac{\partial^{2}}{\partial \theta^{2}}+\frac{1}{\alpha} \frac{\partial}{\partial \alpha}\right)+D_{66}\left(-\frac{2}{\alpha \sin (\varphi)} \frac{\partial^{2}}{\partial \theta \partial \alpha}+\frac{2}{\alpha^{2} \sin ^{2}(\varphi)} \frac{\partial}{\partial \theta}\right)\right)
\end{aligned}
$$

$$
\begin{aligned}
L_{31}= & \frac{1}{\alpha \tan (\varphi)}\left(A_{12}\left(\frac{\partial}{\partial \alpha}\right)+A_{22}\left(\frac{1}{\alpha}\right)+A_{26}\left(\frac{1}{\alpha \sin (\varphi)} \frac{\partial}{\partial \theta}\right)\right) \\
& +\frac{\partial^{2}}{\partial \alpha^{2}}\left(B_{11}\left(\frac{\partial}{\partial \alpha}\right)+B_{12}\left(\frac{1}{\alpha}\right)+B_{16}\left(\frac{1}{\alpha \sin (\varphi)} \frac{\partial}{\partial \theta}\right)\right)+\frac{2}{\alpha \sin (\varphi)} \frac{\partial^{2}}{\partial \theta \partial \alpha}\left(B_{16}\left(\frac{\partial}{\partial \alpha}\right)+B_{26}\left(\frac{1}{\alpha}\right)\right. \\
& \left.+B_{66}\left(\frac{1}{\alpha \sin (\varphi)} \frac{\partial}{\partial \theta}\right)\right)-\frac{1}{\alpha} \frac{\partial}{\partial \alpha}\left(B_{12}\left(\frac{\partial}{\partial \alpha}\right)+B_{22}\left(\frac{1}{\alpha}\right)+B_{26}\left(\frac{1}{\alpha \sin (\varphi)} \frac{\partial}{\partial \theta}\right)\right)+\frac{2}{\alpha} \frac{\partial}{\partial \alpha}\left(B_{11}\left(\frac{\partial}{\partial \alpha}\right)\right. \\
& \left.+B_{12}\left(\frac{1}{\alpha}\right)+B_{16}\left(\frac{1}{\alpha \sin (\varphi)} \frac{\partial}{\partial \theta}\right)\right)+\frac{1}{\alpha^{2} \sin ^{2}(\varphi)} \frac{\partial^{2}}{\partial \theta^{2}}\left(B_{12}\left(\frac{\partial}{\partial \alpha}\right)+B_{22}\left(\frac{1}{\alpha}\right)\right. \\
& \left.+B_{26}\left(\frac{1}{\alpha \sin (\varphi)} \frac{\partial}{\partial \theta}\right)\right)+\frac{2}{\alpha^{2} \sin ^{2}(\varphi)} \frac{\partial}{\partial \theta}\left(B_{16}\left(\frac{\partial}{\partial \alpha}\right)+B_{26}\left(\frac{1}{\alpha}\right)+B_{66}\left(\frac{1}{\alpha \sin (\varphi)} \frac{\partial}{\partial \theta}\right)\right)
\end{aligned}
$$

$$
\begin{aligned}
& L_{32}=-\frac{1}{\alpha \tan (\varphi)}\left(A_{22}\left(\frac{1}{\alpha \sin (\varphi)} \frac{\partial}{\partial \theta}\right)+A_{26}\left(\frac{\partial}{\partial \alpha}-\frac{1}{\alpha}\right)+B_{22}\left(\frac{\cot (\varphi)}{\alpha^{2} \sin ^{2}(\varphi)} \frac{\partial}{\partial \theta}\right)\right. \\
& \left.+B_{26}\left(\frac{\cot (\varphi)}{\alpha} \frac{\partial}{\partial \alpha}-\frac{2 \cot (\varphi)}{\alpha^{2}}\right)\right)+\frac{\partial^{2}}{\partial \alpha^{2}}\left(B_{12}\left(\frac{1}{\alpha \sin (\varphi)} \frac{\partial}{\partial \theta}\right)+B_{16}\left(\frac{\partial}{\partial \alpha}-\frac{1}{\alpha}\right)\right. \\
& \left.+D_{12}\left(\frac{\cot (\varphi)}{\alpha^{2} \sin ^{2}(\varphi)} \frac{\partial}{\partial \theta}\right)+D_{16}\left(\frac{\cot (\varphi)}{\alpha} \frac{\partial}{\partial \alpha}-\frac{2 \cot (\varphi)}{\alpha^{2}}\right)\right)+\frac{2}{\alpha \sin (\varphi)} \frac{\partial^{2}}{\partial \theta \partial \alpha}\left(B_{26}\left(\frac{1}{\alpha \sin (\varphi)} \frac{\partial}{\partial \theta}\right)\right. \\
& \left.+B_{66}\left(\frac{\partial}{\partial \alpha}-\frac{1}{\alpha}\right)+D_{26}\left(\frac{\cot (\varphi)}{\alpha^{2} \sin ^{2}(\varphi)} \frac{\partial}{\partial \theta}\right)+D_{66}\left(\frac{\cot (\varphi)}{\alpha} \frac{\partial}{\partial \alpha}-\frac{2 \cot (\varphi)}{\alpha^{2}}\right)\right)-\frac{1}{\alpha} \frac{\partial}{\partial \alpha}\left(B_{22}\left(\frac{1}{\alpha \sin (\varphi)} \frac{\partial}{\partial \theta}\right)\right. \\
& \left.+B_{26}\left(\frac{\partial}{\partial \alpha}-\frac{1}{\alpha}\right)+D_{22}\left(\frac{\cot (\varphi)}{\alpha^{2} \sin (\varphi)} \frac{\partial}{\partial \theta}\right)+D_{66}\left(\frac{\cot (\varphi)}{\alpha} \frac{\partial}{\partial \alpha}-\frac{2 \cot (\varphi)}{\alpha^{2}}\right)\right)+\frac{2}{\alpha} \frac{\partial}{\partial \alpha}\left(B_{12}\left(\frac{1}{\alpha \sin (\varphi)} \frac{\partial}{\partial \theta}\right)\right. \\
& \left.+B_{16}\left(\frac{\partial}{\partial \alpha}-\frac{1}{\alpha}\right)+D_{12}\left(\frac{\cot (\varphi)}{\alpha^{2} \sin (\varphi)} \frac{\partial}{\partial \theta}\right)+D_{16}\left(\frac{\cot (\varphi)}{\alpha} \frac{\partial}{\partial \alpha}-\frac{2 \cot (\varphi)}{\alpha^{2}}\right)\right)+\frac{1}{\alpha^{2} \sin ^{2}(\varphi)} \frac{\partial^{2}}{\partial \theta^{2}}\left(B_{22}\left(\frac{1}{\alpha \sin (\varphi)} \frac{\partial}{\partial \theta}\right)\right. \\
& \left.+B_{26}\left(\frac{\partial}{\partial \alpha}-\frac{1}{\alpha}\right)+D_{22}\left(\frac{\cot (\varphi)}{\alpha^{2} \sin (\varphi)} \frac{\partial}{\partial \theta}\right)+D_{26}\left(\frac{\cot (\varphi)}{\alpha} \frac{\partial}{\partial \alpha}-\frac{2 \cot (\varphi)}{\alpha^{2}}\right)\right)+\frac{2}{\alpha^{2} \sin (\varphi)} \frac{\partial}{\partial \theta}\left(B_{26}\left(\frac{1}{\alpha \sin (\varphi)} \frac{\partial}{\partial \theta}\right)\right. \\
& \left.+B_{66}\left(\frac{\partial}{\partial \alpha}-\frac{1}{\alpha}\right)+D_{26}\left(\frac{\cot (\varphi)}{\alpha^{2} \sin (\varphi)} \frac{\partial}{\partial \theta}\right)+D_{66}\left(\frac{\cot (\varphi)}{\alpha} \frac{\partial}{\partial \alpha}-\frac{2 \cot (\varphi)}{\alpha^{2}}\right)\right)
\end{aligned}
$$




$$
\begin{aligned}
& L_{33}=-\frac{1}{\alpha \tan (\varphi)}\left(A_{22}\left(\frac{1}{\alpha \tan (\varphi)}\right)-B_{12}\left(\frac{\partial^{2}}{\partial \alpha^{2}}\right)-B_{22}\left(\frac{1}{\alpha^{2} \sin ^{2}(\varphi)} \frac{\partial^{2}}{\partial \theta^{2}}+\frac{1}{\alpha} \frac{\partial}{\partial \alpha}\right)\right. \\
& \left.+B_{26}\left(-\frac{2}{\alpha \sin (\varphi)} \frac{\partial^{2}}{\partial \theta \partial \alpha}+\frac{2}{\alpha^{2} \sin (\varphi)} \frac{\partial}{\partial \theta}\right)\right)+\frac{\partial^{2}}{\partial \alpha^{2}}\left(B_{12}\left(\frac{1}{\alpha \tan (\varphi)}\right)-D_{11}\left(\frac{\partial^{2}}{\partial \alpha^{2}}\right)\right. \\
& \left.-D_{12}\left(\frac{1}{\alpha^{2} \sin ^{2}(\varphi)} \frac{\partial^{2}}{\partial \theta^{2}}+\frac{1}{\alpha} \frac{\partial}{\partial \alpha}\right)+D_{16}\left(-\frac{2}{\alpha \sin (\varphi)} \frac{\partial^{2}}{\partial \theta \partial \alpha}+\frac{2}{\alpha^{2} \sin (\varphi)} \frac{\partial}{\partial \theta}\right)\right) \\
& +\frac{2}{\alpha \sin (\varphi)} \frac{\partial^{2}}{\partial \theta \partial \alpha}\left(B_{26}\left(\frac{1}{\alpha \tan (\varphi)}\right)-D_{16}\left(\frac{\partial^{2}}{\partial \alpha^{2}}\right)-D_{26}\left(\frac{1}{\alpha^{2} \sin ^{2}(\varphi)} \frac{\partial^{2}}{\partial \theta^{2}}+\frac{1}{\alpha} \frac{\partial}{\partial \alpha}\right)\right. \\
& \left.+D_{26}\left(-\frac{2}{\alpha \sin (\varphi)} \frac{\partial^{2}}{\partial \theta \partial \alpha}+\frac{2}{\alpha^{2} \sin (\varphi)} \frac{\partial}{\partial \theta}\right)\right)+\frac{2}{\alpha} \frac{\partial}{\partial \alpha}\left(B_{12}\left(\frac{1}{\alpha \tan (\varphi)}\right)-D_{11}\left(\frac{\partial^{2}}{\partial \alpha^{2}}\right)\right. \\
& \left.-D_{12}\left(\frac{1}{\alpha^{2} \sin ^{2}(\varphi)} \frac{\partial^{2}}{\partial \theta^{2}}+\frac{1}{\alpha} \frac{\partial}{\partial \alpha}\right)+D_{16}\left(-\frac{2}{\alpha \sin (\varphi)} \frac{\partial^{2}}{\partial \theta \partial \alpha}+\frac{2}{\alpha^{2} \sin (\varphi)} \frac{\partial}{\partial \theta}\right)\right) \\
& +\frac{1}{\alpha^{2} \sin ^{2}(\varphi)} \frac{\partial^{2}}{\partial \theta^{2}}\left(B_{22}\left(\frac{1}{\alpha \tan (\varphi)}\right)-D_{12}\left(\frac{\partial^{2}}{\partial \alpha^{2}}\right)-D_{22}\left(\frac{1}{\alpha^{2} \sin ^{2}(\varphi)} \frac{\partial^{2}}{\partial \theta^{2}}+\frac{1}{\alpha} \frac{\partial}{\partial \alpha}\right)\right. \\
& \left.+D_{26}\left(-\frac{2}{\alpha \sin (\varphi)} \frac{\partial^{2}}{\partial \theta \partial \alpha}+\frac{2}{\alpha^{2} \sin (\varphi)} \frac{\partial}{\partial \theta}\right)\right)+\frac{2}{\alpha^{2} \sin (\varphi)} \frac{\partial}{\partial \theta}\left(B_{26}\left(\frac{1}{\alpha \tan (\varphi)}\right)-D_{16}\left(\frac{\partial^{2}}{\partial \alpha^{2}}\right)\right. \\
& \left.-D_{26}\left(\frac{1}{\alpha^{2} \sin ^{2}(\varphi)} \frac{\partial^{2}}{\partial \theta^{2}}+\frac{1}{\alpha} \frac{\partial}{\partial \alpha}\right)+D_{66}\left(-\frac{2}{\alpha \sin (\varphi)} \frac{\partial^{2}}{\partial \theta \partial \alpha}+\frac{2}{\alpha^{2} \sin (\varphi)} \frac{\partial}{\partial \theta}\right)\right)
\end{aligned}
$$

\section{References}

[1] D.J. Wilkins, C.W. Bert, D.M. Egle, Free vibrations of orthotropic sandwich conical shells with various boundary conditions, J. Sound. Vib. 13 (1970) 211-228

[2] H. Ramsey, Contrast between elastic and plastic buckling of axially compressed conical shells with edge constraint, Int. J. Mech. Sci. 20 (1978) 37-46

[3] T. Irie, G. Yamada, Y. Muramoto, Free vibration of joined conical-cylindrical shells, J. Sound. Vib. 95 (1984) 31-39

[4] H. Zhou, D. Liu, Mechanical analysis of the elastic buckling of an orthogonal anisotropic circular conical shell under uniform external pressure, Int. J. Pres. Ves. Pip. 48 (1991) 111-122

[5] D.P. Thambiratnam, Y. Zhuge, Axisymmetric free vibration analysis of conical shells, Eng. Struct. 15 (1993) $83-89$

[6] C. Shu, An efficient approach for free vibration analysis of conical shells, Int. J. Mech. Sci. 38 (1996) 935-949

[7] A. Spagnoli, M.K. Chryssanthopoulos, Elastic buckling and post buckling behaviour of widely-stiffened conical shells under axial compression, Eng. Struct. 21 (1999) 845-855

[8] K.Y. Lam, L. Hua, On free vibration of a rotating truncated circular orthotropic conical shell, Compos. Part B: Eng. 30 (1999) 135-144

[9] A. Spagnoli, Different buckling modes in axially stiffened conical shells, Eng. Struct. 23 (2001) 957-965

[10] H.T. Hu, S.C. Ou, Maximization of the fundamental frequencies of laminated truncated conical shells with respect to fiber orientations, Compos. Struct. 52 (2001) 265-275

[11] C.P. Wu, C.Y. Lee, Differential quadrature solution for the free vibration analysis of laminated conical shells with variable stiffness, Int. J. Mech. Sci. 43 (2001) 1853-1869

[12] X.X. Hu, T. Sakiyama, H. Matsuda, C. Morita, Vibration of twisted laminated composite conical shells, Int. J. Mech. Sci. 44 (2002) 1521-1541
[13] Y. Goldfeld, J. Arbocz, A. Rothwell, Design and optimization of laminated conical shells for buckling, Thin Wall. Struct. 43 (2005) 107-133

[14] C.T.F. Ross, A.P.F. Little, K.A. Adeniyi, Plastic buckling of ring-stiffened conical shells under external hydrostatic pressure, Ocean Eng. 32 (2005) 21-36

[15] O. Civalek, An efficient method for free vibration analysis of rotating truncated conical shells, Int. J. Pres. Ves. Pip. 83 (2006) $1-12$

[16] N.K. Gupta, N. Mohamed Sheriff, R. Velmurugan, A study on buckling of thin conical frusta under axial loads, Thin Walled. Struct. 44 (2006) 986-996

[17] S. Liang, H.L. Chen, T. Chen, M.Y. Wang, The natural vibration of a symmetric cross-ply laminated composite conical-plate shell, Compos. Struct. 80 (2007) 265-278

[18] V. Tripathi, B.N. Singh, K.K. Shukla, Free vibration of laminated composite conical shells with random material properties, Compos. Struct. 81 (2007) 96-104

[19] O. Civalek, Numerical analysis of free vibrations of laminated composite conical and cylindrical shells: Discrete singular convolution (DSC) approach, J. Comput. Appl. Math. 205 (2007) 251-271

[20] A.H. Sofiyev, N. Kuruoglu, M. Turkmen, Buckling of FGM hybrid truncated conical shells subjected to hydrostatic pressure, Thin Walled. Struct. 47 (2009) 61-72

[21] A.H. Sofiyev, K.A. Korkmaz, Z. Mammadov, M. Kamanli, The vibration and buckling of freely supported non-homogeneous orthotropic conical shells subjected to different uniform pressures, Int. J. Pres. Ves. Pip. 86 (2009) 661-668

[22] A.H. Sofiyev, The vibration and stability behavior of freely supported FGM conical shells subjected to external pressure, Compos. Struct. 89 (2009) 356-366 
[23] A.H. Sofiyev, N. Kuruoglu, H.M. Halilov, The vibration and stability of non-homogeneous orthotropic conical shells with clamped edges subjected to uniform external pressures, Appl. Math. Model. 34 (2010) 1807-1822

[24] A.H. Sofiyev, Influence of the initial imperfection on the non-linear buckling response of FGM truncated conical shells, Int. J. Mech. Sci. 53 (2011) 753-761

[25] J.B. Lachut, On elasti-plastic buckling of cones, Thin Walled. Struct. 49 (2011) 45-52

[26] A.H. Sofiyev, Non-linear buckling behavior of FGM truncated conical shells subjected to axial load, Int. J. Nonlinear Mech. 46 (2011) 711-719

[27] A.H. Sofiyev, N.K. Kuruoglu, The non-linear buckling analysis of cross-ply laminated orthotropic truncated conical shells, Compos. Struct. 93 (2011) 3006-3012

[28] M. Nedelcu, GBT formulation to analyses of the buckling behaviour of isotropic conical shells, Thin Walled. Struct. 49 (2011) 812-818

[29] A. Vlot, Historical overview In: Fibre metal laminates, an introduction, Dordrecht, Kluwer Academic Publishers, 2001
[30] M.S. Qatu, Vibration of laminated shells and plates, San Diego CA, Elsevier, 2004

[31] W. Soedel, Vibrations of shells and plates New York, Marcel Dekker, 1993

[32] C.T. Loy, K.Y. Lam, Vibration of Cylindrical Shells with Ring Support, Int. J. Mech. Sci. 39 (1997) 445-471

[33] T. Irie, G. Yamada, K. Tanaka, Natural frequencies of truncated conical shells, J. Sound. Vib. 92 (1984) 447-453

[34] F.M. Li, K. Kishimoto, W.H. Huang, The calculations of natural frequencies and forced vibration responses of conical shell using the Rayleigh-Ritz method, Mech. Res. Commun. 36 (2009) 595-602

[35] C. Shu, Free vibration analysis of composite laminated conical shells by generalized differential quadrature, J. Sound. Vib. 194 (1996) 587-604 